\title{
Damage to the blood-brain barrier and activation of neuroinflammation by focal cerebral ischemia under hyperglycemic condition
}

\author{
YONGZHEN GUO ${ }^{1,2^{*}}$, LINGDI DONG ${ }^{1 *}$, AO GONG $^{1 *}$, JINGWEN ZHANG $^{1}$, LI JING $^{1}$, \\ TOMAS DING ${ }^{3}$, PING-AN ANDY $\mathrm{LI}^{3}$ and JIAN-ZHONG ZHANG ${ }^{1}$
}

${ }^{1}$ Department of Pathology, School of Basic Medical Science, Ningxia Medical University,

Ningxia Key Laboratory of Cerebrocranial Diseases, Incubation Base of National Key Laboratory, Yinchuan, Ningxia 750004; ${ }^{2}$ Department of Pathology, The Third Affiliated Hospital of Zhengzhou University, Zhengzhou, Henan 450052, P.R. China; ${ }^{3}$ Department of Pharmaceutical Sciences, Biomanufacturing Research Institute and Technological Enterprise, College of Health and Sciences, North Carolina Central University, Durham, NC 27707, USA

Received December 26, 2020; Accepted April 28, 2021

DOI: $10.3892 /$ ijmm.2021.4975

\begin{abstract}
Hyperglycemia aggravates brain damage caused by cerebral ischemia/reperfusion (I/R) and increases the permeability of the blood-brain barrier (BBB). However, there are relatively few studies on morphological changes of the BBB. The present study aimed to investigate the effect of hyperglycemia on BBB morphological changes following cerebral I/R injury. Streptozotocin-induced hyperglycemic and citrate-buffered saline-injected normoglycemic rats were subjected to $30 \mathrm{~min}$ middle cerebral artery occlusion. Neurological deficits were evaluated. Brain infarct volume was assessed by 2,3,5-triphenyltetrazolium chloride staining and BBB integrity was evaluated by Evans blue and IgG extravasation following $24 \mathrm{~h}$ reperfusion. Changes in tight junctions (TJ) and basement membrane (BM) proteins (claudin, occludin and zonula occludens-1) were examined using immunohistochemistry and western blotting. Astrocytes, microglial cells and neutrophils were labeled with specific antibodies for immunohistochemistry
\end{abstract}

Correspondence to: Professor Jian-Zhong Zhang, Department of Pathology, School of Basic Medical Science, Ningxia Medical University, Ningxia Key Laboratory of Cerebrocranial Diseases, Incubation Base of National Key Laboratory, 1160 Shengli Street, Yinchuan, Ningxia 750004, P.R. China

E-mail: zhangjz@nxmu.edu.cn

Professor Ping-An Andy Li, Department of Pharmaceutical Sciences, Biomanufacturing Research Institute and Technological Enterprise, College of Health and Sciences, North Carolina Central University, 1801 Fayetteville Street, Durham, NC 27707, USA

E-mail: tedaiea@aol.com

*Contributed equally

Key words: blood-brain barrier, cerebral ischemia, diabetes, hyperglycemia, neurovascular unit, reperfusion injury after 1, 3 and 7 days of reperfusion. Hyperglycemia increased extravasations of Evan's blue and $\mathrm{IgG}$ and aggravated damage to $\mathrm{TJ}$ and $\mathrm{BM}$ proteins following I/R injury. Furthermore, hyperglycemia suppressed astrocyte activation and damaged astrocytic endfeet surrounding cerebral blood vessels following I/R. Hyperglycemia inhibited microglia activation and proliferation and increased neutrophil infiltration in the brain. It was concluded that hyperglycemia-induced BBB leakage following $\mathrm{I} / \mathrm{R}$ might be caused by damage to TJ and BM proteins and astrocytic endfeet. Furthermore, suppression of microglial cells and increased neutrophil infiltration to the brain may contribute to the detrimental effects of pre-ischemic hyperglycemia on the outcome of cerebral ischemic stroke.

\section{Introduction}

Hyperglycemia is a major risk factor for ischemic stroke in the clinic and increases the risk of mortality and morbidity in patients suffering from stroke (1). The Emerging Risk Factors Collaboration showed that the adjusted hazard ratio for patients with with diabetes was 2.27 (1.95-2.65) for ischemic stroke (2). Animal studies have confirmed that hyperglycemia enlarges cerebral infarction volume and causes hemorrhagic transformation of the infarct area, resulting in a poorer prognosis $(3,4)$. The mechanism of hyperglycemia aggravated ischemic brain injury may involve lactic acidosis, oxidative stress and activation of cell death pathways $(5,6)$. Our previous studies have demonstrated that imbalance of mitochondrial fission/fusion and decreased mitochondrial biogenesis contribute to hyperglycemia-enhanced ischemic brain damage (7-9).

The neurovascular unit (NVU), with the blood-brain barrier (BBB) as its core, emphasizes the close link between brain tissue and its blood supply (10). The BBB is a structural and functional barrier between the central nervous system (CNS) and the systemic blood circulation. It prevents harmful substances from entering the brain parenchyma and regulates the transport of water, ions and macromolecules, thus maintaining the 
stability of brain homeostasis (11). The major components of the BBB include vascular endothelial cells interconnected with tight junctions (TJ), basement membrane (BM) and astrocytic endfeet. TJs are located between adjacent vascular endothelial cells, where they play a key role in limiting paracellular permeability, thus regulating permeability of the BBB. Three types of transmembrane proteins primarily constitute the TJ: Claudin, occludin and zonula occluden-1 (ZO-1) (12). Claudin is the principal component of the TJ complex and is involved in selective permeability and cellular polarization (13). It is considered to be the most important regulator of cerebrovascular endothelial cell permeability (14). There are different subtypes of claudin. Claudin-5 is the primary transmembrane protein that forms the BBB (15). Miyamori et al (16) observed that in the late stage of cerebral ischemia/reperfusion (I/R), claudin-5 migrates from endothelial cells to surrounding astrocytes, which disrupts the BBB. Occludin is considered as a staple of TJs between blood endothelial cells. Occludin expression in cerebrovascular endothelial cells is suppressed and integrity of the BBB is compromised following cerebral I/R (17). This suggests that decreased expression of occludin can be used as an indicator of BBB disruption. ZO-1 is the first confirmed $\mathrm{TJ}$ adhesion protein (18) and is used as a marker for BBB damage. It connects transmembrane proteins (occludin and claudins) with cytoskeletal proteins on the inner side of the cell (such as actin), thus serving a key role in TJ (18). During cerebral ischemia/reperfusion (I/R), expression of ZO-1 decreases significantly, resulting in degradation of TJ, increased BBB permeability and formation of vasogenic cerebral edema (19). BM is located at the basal side of epithelial cells and is composed of laminin, collagen IV, nidogen and heparan sulfate proteoglycans (20-22). The role of BM ranges from providing structural support to modulating molecular signaling and maintaining normal function of the $\operatorname{BBB}(23,24)$. Loss of BM occurs soon after the onset of ischemia (25), or as early as $10 \mathrm{~min}$ post-reperfusion in a middle cerebral artery occlusion (MCAO) model (26). Astrocytic endfeet cover almost the entire surface of intraparenchymal capillaries in the adult brain and are involved in the formation and maintenance of the BBB (27). In ischemic brain, astrocytes are activated, reflected by increased numbers of dendrites and enlargement of the cell body (28). Moreover, swelling of astrocytic endfeet is one of the earliest morphological events following ischemic stroke (29). Although several studies have shown that hyperglycemia significantly increases brain edema and BBB dysfunction, as shown by Evans blue leakage following I/R injury (30-32), detailed morphological study of the BBB has not previously been performed.

Microglia, macrophages, neutrophils and T lymphocytes are the most well-studied immune cells in the CNS that interact directly or indirectly with $\mathrm{BBB}$ components and affect the integrity of the BBB following ischemia (33). Microglial cells, the resident immune cells in the brain, are among the first responders to ischemia, followed by neutrophil influx and infiltration of peripheral macrophages, lymphocytes and dendritic cells $(34,35)$. Activated microglia serve dual role in the BBB and ischemic brain damage. On one hand, they produce excessive cytokines and chemokines that overexpress cell adhesion molecules and promote leukocyte infiltration (36). On the other hand, activated microglia may be beneficial to the injured brain by phagocytizing cell debris and inhibiting the inflammatory response (33). In addition, damage to the BBB permits extravasation of neutrophils to the brain parenchyma, which increases the release of deleterious inflammatory mediators. Accumulation of neutrophils, along with proinflammatory mediators and proteases, also potentiates junction disassembly, endothelial malfunction, and extracellular matrix degradation, resulting in irreversible BBB disruption (37). The effect of hyperglycemia on activation of microglia and neutrophil infiltration is in need of further investigation.

The aim of the present study was to investigate the effect of preischemic hyperglycemia on key components of the BBB following I/R injury in the rat brain. To this end, streptozotocin (STZ)-induced hyperglycemic (HG) rats were subjected to 30 min MCAO, followed by 1, 3 or 7 days reperfusion. Levels of TJ and BM-associated proteins. as well as changes in astrocytes and microglia and neutrophil infiltration, were assessed using immunohistochemistry (IHC) and/or western blotting.

\section{Materials and methods}

Animals. A total of 95 male 8-week-old Sprague-Dawley rats (weight, 200-230 g) were provided by the Experimental Animal Center of Ningxia Medical University (Yinchuan China). The animals were reared in a specific-pathogen-free environment with a 12-h light/dark cycle and free access to water and food, the temperature and humidity were controlled $\left(23^{\circ} \mathrm{C}, 55 \%\right.$ humidity). Animal procedures were performed in strict accordance with the Chinese Laboratory Animal Use Regulation and were approved by the Institutional Animal Care and Use Committee of Ningxia Medical University.

STZ-induced diabetic hyperglycemia. A total of 95 rats were fasted for $12 \mathrm{~h}$ and intraperitoneally injected with STZ $(60 \mathrm{mg} / \mathrm{kg})$ freshly dissolved in citrate buffer $(\mathrm{pH} 4.5)$ or an equal amount of solvent as a control. Blood glucose levels were measured 3 days after STZ injection. Rats with blood glucose $>16.7 \mathrm{mM}$ were placed in the $\mathrm{HG}$ group. The rat body weight and blood glucose levels were measured once/week for 4 weeks Cerebral ischemia was induced after 4 weeks in STZ-induced diabetic and citrate buffer-injected normoglycemic (NG) rats. Animals in the NG and $\mathrm{HG}$ groups were further divided into Sham, 1-, 3- and 7 day I/R (I/R 1, 3 and 7 day) subgroups. Animal groups and number of animals in each group are given in Table I.

$I / R$ model. MCAO was induced for $30 \mathrm{~min}$ by inserting an intraluminal filament through the right common carotid artery and advancing to the internal carotid artery and the base of the middle cerebral artery. Reperfusion was achieved by withdrawing the filament after $30 \mathrm{~min}$ occlusion. Neurological defects were scored at 1 day post-I/R according to the modified Neurological Severity Score (mNSS), which primarily assesses sensory, motor, reflex, balance and muscle tone (35). The higher the score, the more serious the neurological deficit. Normal rats have score 0 , and mild, moderate, and severe injuries scores are 1-6, 7-12 and 3-18, respectively. The deficit score was assessed 3 times in each rat and the mean value was calculated. At the predetermined experimental end-points, the rats (including the sham group) were euthanized and the brain 
Table I. Animal groups and numbers.

A, Normoglycemic

\begin{tabular}{lccc}
\hline Group & TTC staining & IHC & WB \\
\hline Sham & 5 & 5 & 3 \\
I/R 1 day & 6 & 7 & 4 \\
I/R 3 days & 0 & 6 & 4 \\
I/R 7 days & 0 & 5 & 3 \\
\hline
\end{tabular}

\section{B, Hyperglycemic}

\begin{tabular}{lccc}
\hline Group & TTC staining & IHC & WB \\
\hline Sham & 0 & 5 & 4 \\
I/R 1 day & 5 & 9 & 4 \\
I/R 3 days & 0 & 6 & 5 \\
I/R 7 days & 0 & 5 & 4 \\
\hline
\end{tabular}

TTC, 2,3,5-triphenyltetrazolium chloride; IHC, immunohistochemistry; WB, western blotting; I/R, ischemia/reperfusion.

was extracted. The brain samples used for morphological studies were fixed in $4 \%$ paraformaldehyde for $4 \mathrm{~h}$ at room temperature and embedded in paraffin. The brain samples used for western blot analysis were frozen in liquid nitrogen and stored in a $-80^{\circ} \mathrm{C}$ freezer. Brain edema was evaluated by dry-wet weight ratio as follows: W/D ratio=(wet weight-dry we ight)/dry weight

\section{2,3,5-Triphenyltetrazolium chloride (TTC) \& Evans blue} staining. The cerebral infarct volume and integrity of the BBB were assessed by TTC and Evans blue staining, respectively, as previously described (38). Briefly, Evans blue dye (2\%; $4 \mathrm{ml} / \mathrm{kg}$ ) was injected into the left femoral vein and allowed to circulate for $60 \mathrm{sec}$ before the rats were euthanized with $1 \%$ pentobarbital $(40 \mathrm{mg} / \mathrm{kg})$ and perfused transcardially with PBS for $60 \mathrm{sec}$ at $37^{\circ} \mathrm{C}$. The brains were removed, washed with PBS and kept at $-20^{\circ} \mathrm{C}$ for $30 \mathrm{~min}$ before sectioning. Coronal sections $(2 \mathrm{~mm})$ were cut in a rat brain matrix and stained with $2 \%$ TTC (Amresco, LLC) for $30 \mathrm{~min}$ at $37^{\circ} \mathrm{C}$. Following staining, the brain slices were fixed with $4 \%$ paraformaldehyde for $10 \mathrm{~min}$ at $37^{\circ} \mathrm{C}$ to conserve the area stained by TTC. The stained brain sections were digitally photographed using a digital camera (PowerShot G7 X Mark II Canon, Inc.). The infarcted area (white) and BBB disruption (blue) of each brain section was measured using Image-Pro Plus 6.0 software (Media Cybernetics, Inc.). The infarcted volume and the BBB disrupted area were calculated according to the formula: $100 \% \mathrm{x}$ (ipsilateral volume-contralateral volume)/contralateral volume.

Immunohistochemistry (IHC). IHC staining was performed as described previously (9). Briefly, the $4-\mu$ m-thick brain sections were de-paraffinized, rehydrated (in descending alcohol series) and washed (in PBS). Antigen retrieval was performed using a high-temperature $\left(120^{\circ} \mathrm{C}\right)$ and high-pressure antigen repairing method in sodium citrate buffer ( $\mathrm{pH}$ 6.0). The sections were then blocked with $3 \% \mathrm{H}_{2} \mathrm{O}_{2}$ and $10 \%$ normal goat serum (OriGene Technologies, Inc.) in PBS for $30 \mathrm{~min}$ at $37^{\circ} \mathrm{C}$. The sections were incubated overnight at $4^{\circ} \mathrm{C}$ with primary antibodies against von Willebrand Factor (vWF; 1:300; cat. no. ab6994; Abcam), claudin-5 (1:100; cat. no. ab15106; Abcam), ZO-1 (1:200; cat. no. ab216880; Abcam), occludin (1:200; cat. no. ab216327; Abcam), laminin (1:200; cat. no. ab11575; Abcam), collagen IV (1:400; cat. no. ab6586; Abcam), glial fibrillary acidic protein (GFAP; 1:200; cat. no. 3670S; Cell Signaling Technology, Inc.) and anti-neutrophil antibody (NIMP-R14; 1:50; cat. no. ab2557; Abcam). The sections were rewarmed for $60 \mathrm{~min}$ at room temperature and then incubated with horseradish peroxidase (HRP)-conjugated secondary antibody (1:300; cat. no. TA130003, OriGene Technologies, Inc.) for $30 \mathrm{~min}$ at $37^{\circ} \mathrm{C}$. Color reaction was developed by 3,3'-diaminobenzidine (DAB) (1:20) incubation for $5 \mathrm{~min}$ and counterstained with hematoxylin for $1 \mathrm{~min}$, both at $37^{\circ} \mathrm{C}$.

For IgG staining, after being de-paraffinized, rehydrated and blocked as aforementioned, the sections were incubated with HRP-conjugated Affinipure Goat Anti-Mouse IgG (H+L; 1:2,000; cat. no. SA00001-1; ProteinTech Group, Inc.) for $2 \mathrm{~h}$ at $37^{\circ} \mathrm{C}$. The sections were washed, and color reaction was developed by DAB (1:20) incubation for $5 \mathrm{~min}$ and counterstained with hematoxylin for $1 \mathrm{~min}$ at $37^{\circ} \mathrm{C}$. The images were captured using a light microscope (cat. no. VM1000; Motic Incorporation, Ltd.; magnification, $\mathrm{x} 40$ ), and the IgG-positive areas were measured using Image-Pro-Plus 6.0 software (Media Cybernetics, Inc.).

The immunofluorescent double labeling of vWF (1:200; cat. no. ab6994; Abcam), with astrocyte marker GFAP (1:200; cat. no. 3670S; Cell Signaling Technology, Inc.) and double labeling of proliferating cell nuclear antigen (PCNA; 1:400; cat. no. ab29; Abcam) with the microglia marker ionized calcium-binding adaptor molecule (IBA1) (1:200; cat. no. ab5076; Abcam) were achieved by incubating the two primary antibodies with brain sections separately for $4 \mathrm{~h}$ at $37^{\circ} \mathrm{C}$ and then with a mixture of two different fluorescence-conjugated secondary antibodies for $2 \mathrm{~h}$ at $37^{\circ} \mathrm{C}$. The specimens were mounted with Antifade Polyvinylpyrrolidone Mounting Medium (Vector Informatik $\mathrm{GmbH}$ ) containing DAPI and then examined under a fluorescence confocal-scanning microscope (cat. no. FV1000; Olympus Corporation; magnification, $\mathrm{x} 400$ ).

Morphological analysis. Following immunostaining, astrocyte and microglia morphology were evaluated using software (ImageJ; version 2.0.0-rc-69/1.52p; National Institutes of Health). Analysis of dendrite branching features, including number of endpoints, total segment length and maximum branch length, was performed using the plugin Analyze Skeleton (fiji.sc/AnalyzeSkeleton) (39). Briefly, images were converted to 8-bit gray type and filtered with Fast Fourier Transform bandpass filter plugin (imagej.nih. gov/ij/plugins/fft-filter.html). The brightness and contrast were adjusted to show cell morphology and noise was removed. The images were then skeletonized and the number of endpoints, total segment length and maximum branch length were measured automatically using the Analyze Skeleton plugin. The area of $\mathrm{IBA}^{+}$stained cells and circularity index 

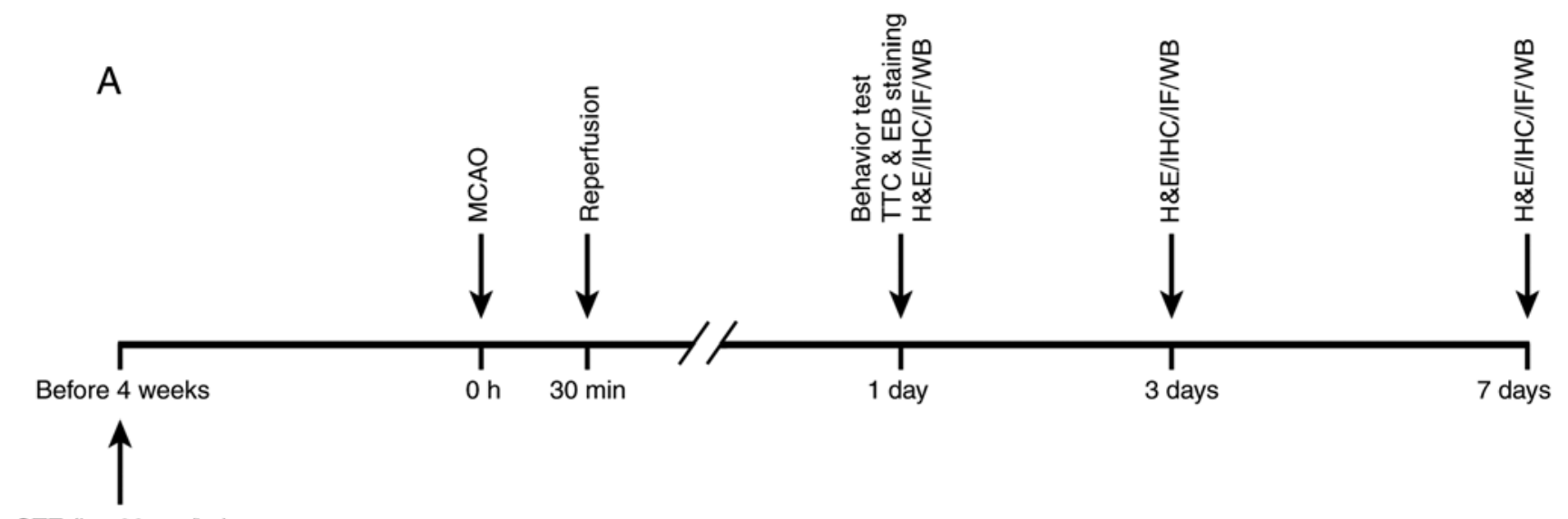

STZ (i.p. $60 \mathrm{mg} / \mathrm{kg}$ )
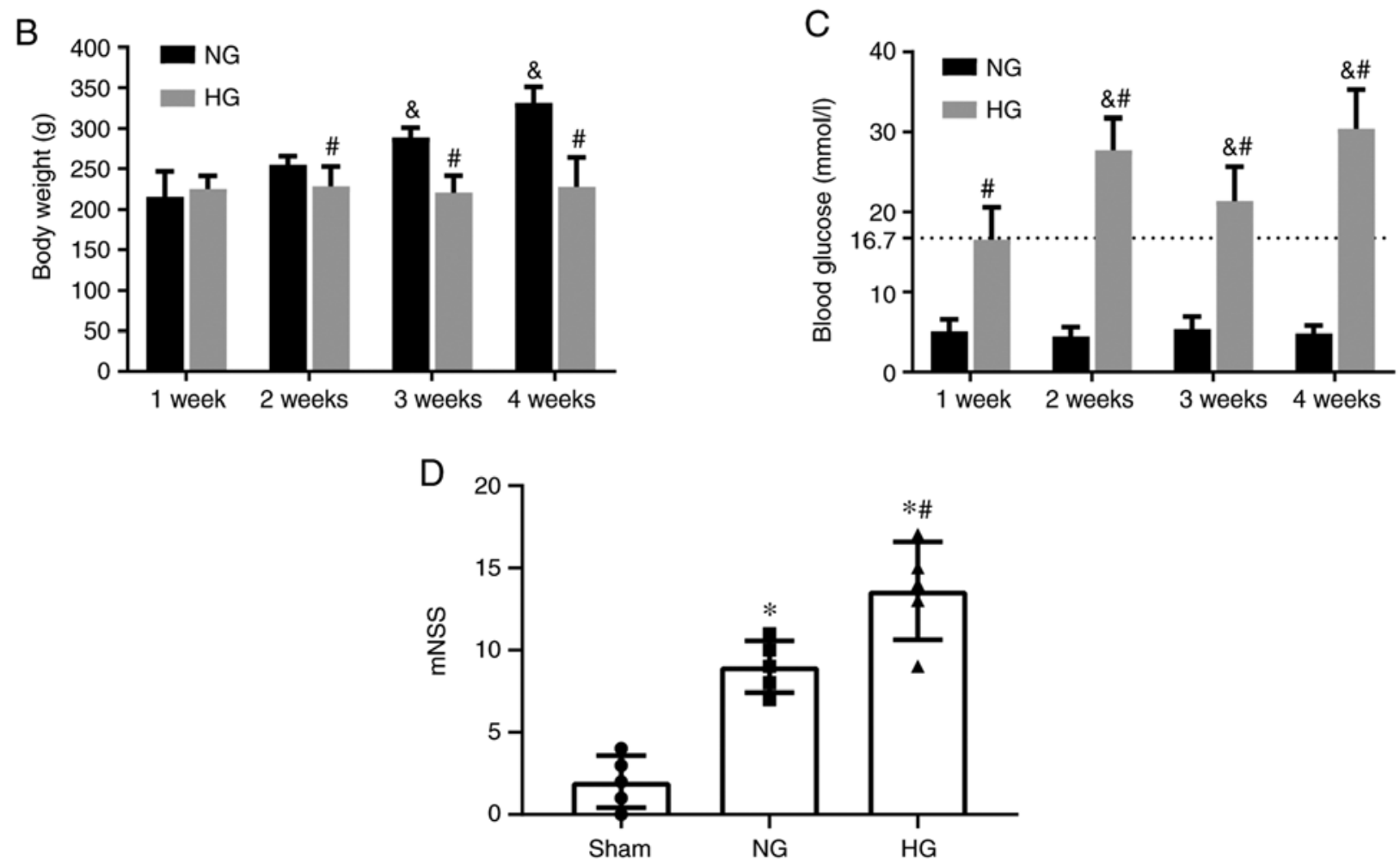

Figure 1. Schematic diagram and physiological data (A) Schematic diagram of experimental design. Changes in (B) body weight, (C) blood glucose level and (D) neurological deficit following cerebral I/R in NG and HG animals. Hyperglycemia was induced by STZ injection and neurological deficit was assessed using the $\mathrm{mNSS}$ at 1 day post-reperfusion following $30 \mathrm{~min}$ MCAO. $\mathrm{n}=5$ /group. ${ }^{\circledR} \mathrm{P}<0.05$ vs. 1 week, ${ }^{*} \mathrm{P}<0.05$ vs. Sham, ${ }^{\text {}} \mathrm{P}<0.05$ vs. NG. NG, normoglycemic; HG, hyperglycemic; STZ, streptozotocin; MCAO, middle cerebral artery occlusion; i.p., intraperitoneal; H\&E, hematoxylin and eosin; IHC, immunohistochemistry; IF, immunofluorescence; WB, western blotting; mNSS, modified neurological severity score; TTC, 2,3,5-triphenyltetrazolium chloride; EB, Evans blue.

(CI) were measured using the plugin Shape Descriptors (40). The CI parameter was calculated by the Shape Descriptors plugin as follows: Area $=\left[4 p(\right.$ area $\left.) /(\text { perimeter })^{2}\right](41)$. The numbers of $\mathrm{vWF}^{+}, \mathrm{GFAP}^{+}$and $\mathrm{IBA}^{+}$cells were counted using Image-Pro-Plus 6.0 software (Media Cybernetics, Inc.).

Western blotting. Following reperfusion, ipsilateral brain tissue was homogenized in RIPA lysis buffer containing protease inhibitors (Nanjing KeyGen Biotech Co., Ltd.). The protein concentration of the supernatants $\left(24,148.8 \times \mathrm{g}, 5 \mathrm{~min}, 4^{\circ} \mathrm{C}\right)$ was measured using a BCA Protein Quantitation Assay (Nanjing KeyGen Biotech Co., Ltd.). Equal amounts of protein $(30 \mu \mathrm{g})$ were loaded onto $8-10 \%$ sodium dodecyl sulfate-polyacrylamide gels. Following electrophoresis, bands were transferred onto PVDF membranes. After being blocked with 5\% BSA (OriGene Technologies, Inc.) for $2 \mathrm{hat} 37^{\circ} \mathrm{C}$, the membranes were incubated overnight at $4^{\circ} \mathrm{C}$ using the aforementioned primary antibodies against claudin-5, ZO-1, occludin, laminin and collagen IV or TNF- $\alpha$ (1:1,000; cat. no. 17590-1-AP; ProteinTech Group, Inc.), IL-1 $\beta$ (1:500; cat. no. ab200478; Abcam) or IL-6 (1:2,000; cat. no. 66146-1-Ig; ProteinTech Group, Inc.). The membranes were washed and incubated with HRP-conjugated secondary antibody (1:2,000; cat. no. SA00013-2; ProteinTech Group, Inc.) for $2 \mathrm{~h}$ at room temperature. Visualization was performed using ECL kit (cat. no. 34577; Thermo Fisher Scientific, Inc.). The immunoblots were visualized using a computerized image 
A

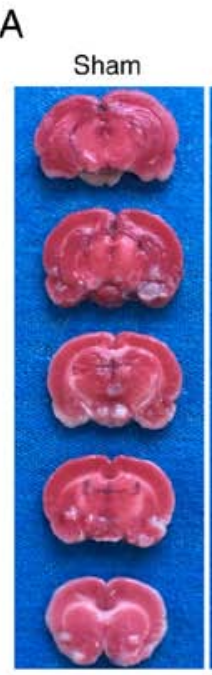

E

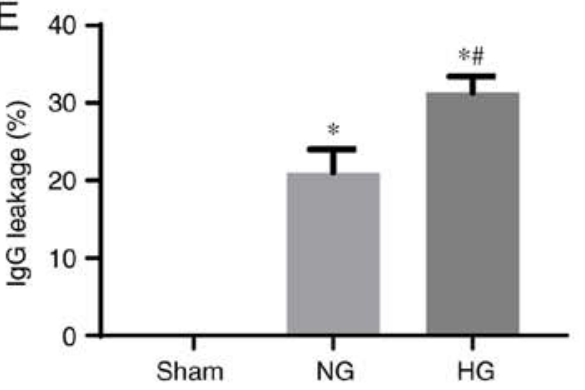

B

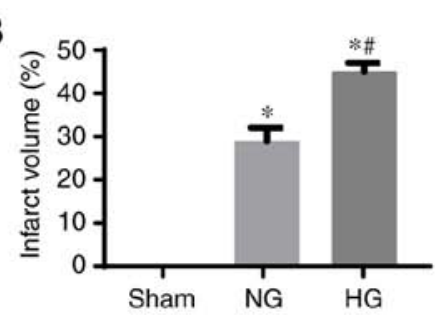

C

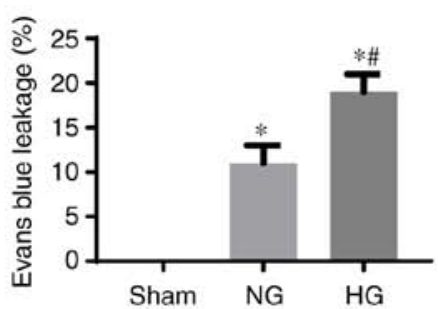

D

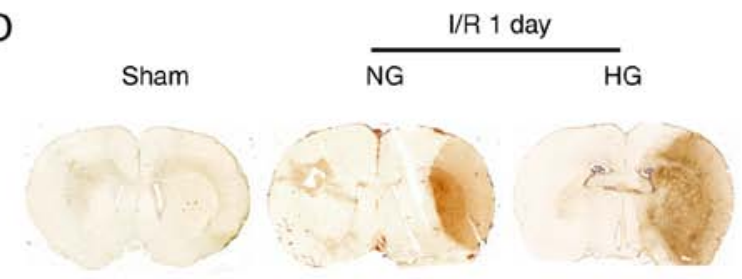

F

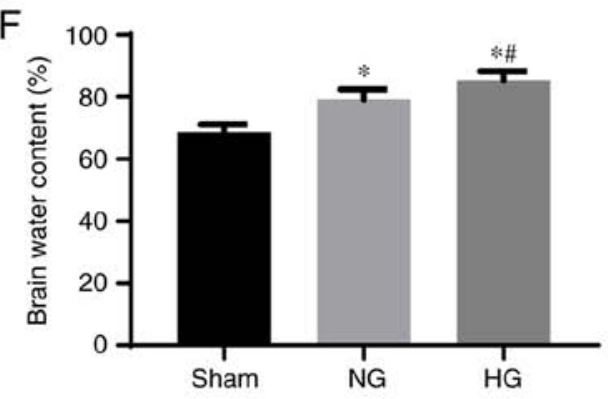

G

Sham

I/R 1 day

I/R 3 days

I/R 7 days

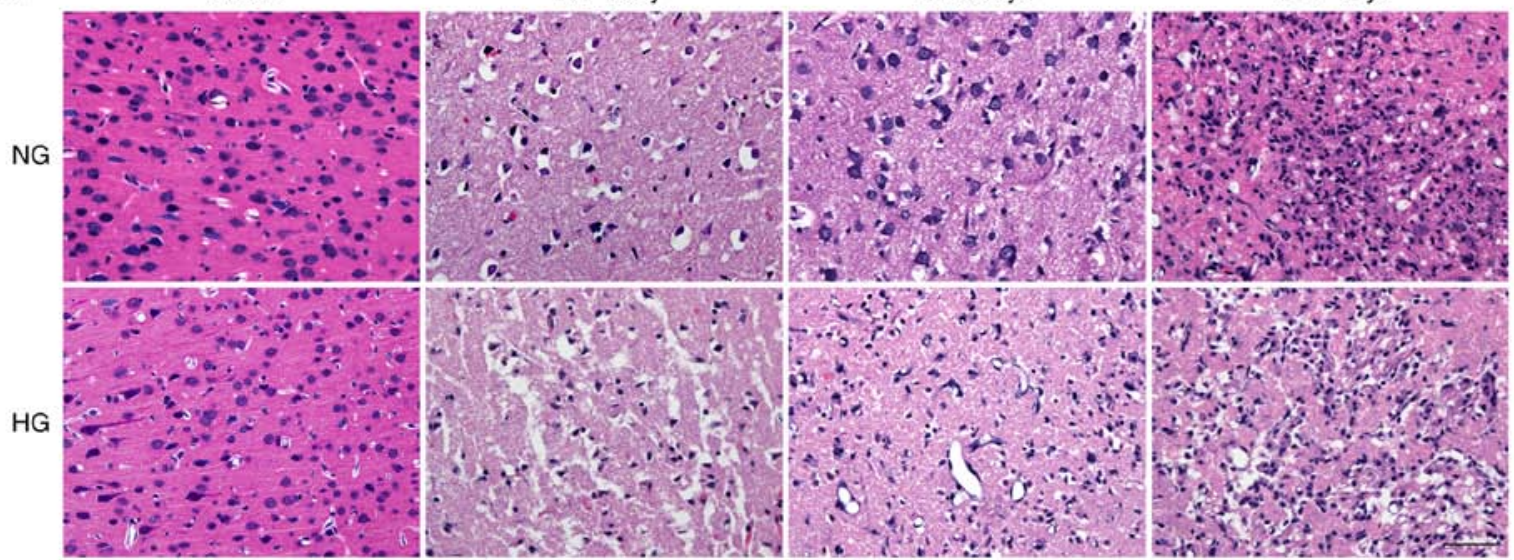

Figure 2. Permeability of the BBB following I/R. (A) Representative brain sections stained with 2,3,5-triphenyltetrazolium chloride and Evans blue at 1 day after I/R. Pale areas show infarct volume; blue areas show Evans blue extravasation. Quantitative summary of (B) infarct volume and (C) Evans blue extravasation (BBB damage) area. (D) Representative brain slices stained with anti-IgG antibody at 1 day post-I/R. (E) Quantitative summary of IgG extravasation area expressed as percent of the whole brain section area. (F) Quantitative summary of brain water content through wet and dry brain weight ratio. (G) Representative photomicrographs of hematoxylin and eosin-stained cortex. Scale bar, $50 \mu \mathrm{m}$. $\mathrm{n}=5 /$ group. ${ }^{*} \mathrm{P}<0.05$ vs. Sham, " $\mathrm{P}<0.05$ vs. NG. BBB, blood-brain barrier; I/R, ischemia/reperfusion; NG, normoglycemic; $\mathrm{HG}$, hyperglycemic.

analysis system (Amersham Imager 600; Cytiva), and the results were expressed as the ratio of corresponding protein to $\beta$-actin (1:5,000; cat. no. SA00001-7L; ProteinTech Group, Inc.).

Statistical analysis. Data are presented as the mean \pm SEM $(n=3)$. Data were analyzed by one- or two-way ANOVA followed by post hoc Tukey's test. All statistical analysis was performed using the SPSS 22.00 software (IBM Corp.). P $<0.05$ was considered to indicate a statistically significant difference.

\section{Results}

Hyperglycemia enhances neurological deficit following cerebral I/R injury. The experimental paradigm is presented in Fig. 1A. The rat body weight and blood glucose levels were measured once/week for 4 weeks following STZ injection. Weight of NG animals increased from $215.7 \pm 31$ to $331.2 \pm 20 \mathrm{~g}$ after 4 weeks; HG animals did not gain weight during the 4 -week period. As a result, the body weight of HG animals in weeks 2-4 were significantly lower (Fig. 1B). The blood 


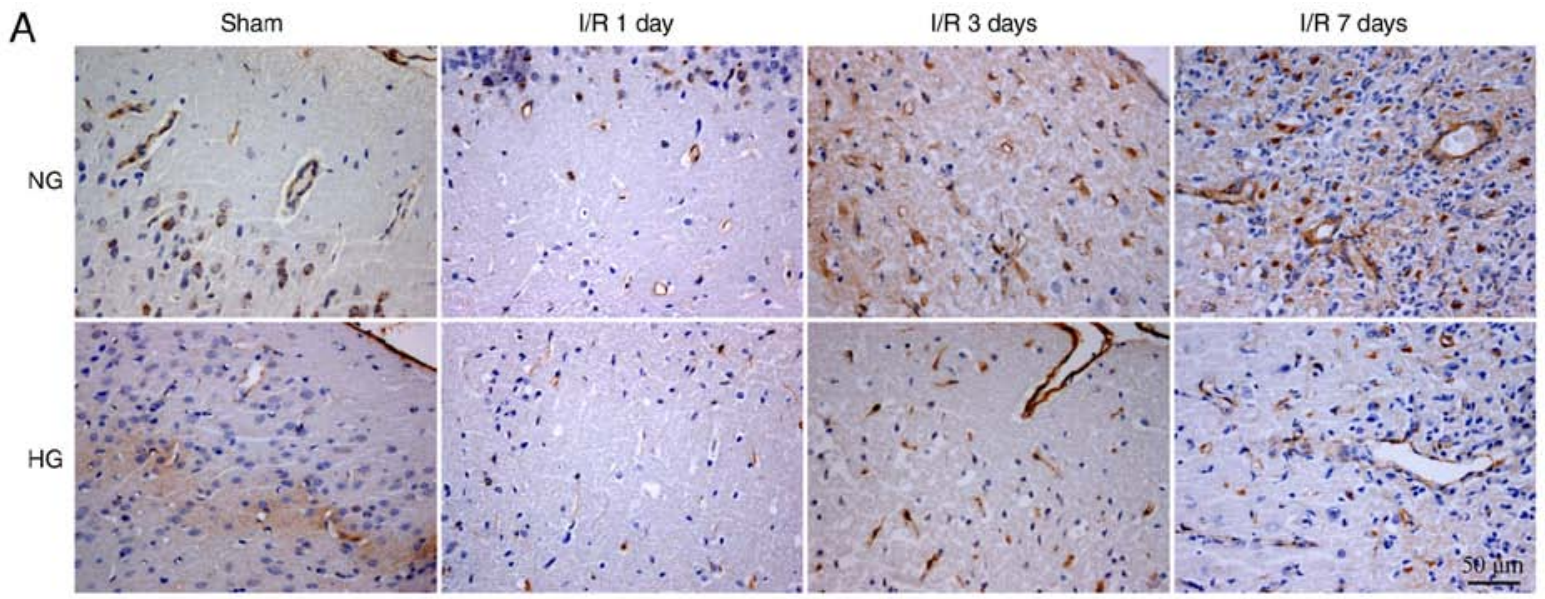

B

B Sham

I/R 1 day

I/R 3 days

I/R 7 days

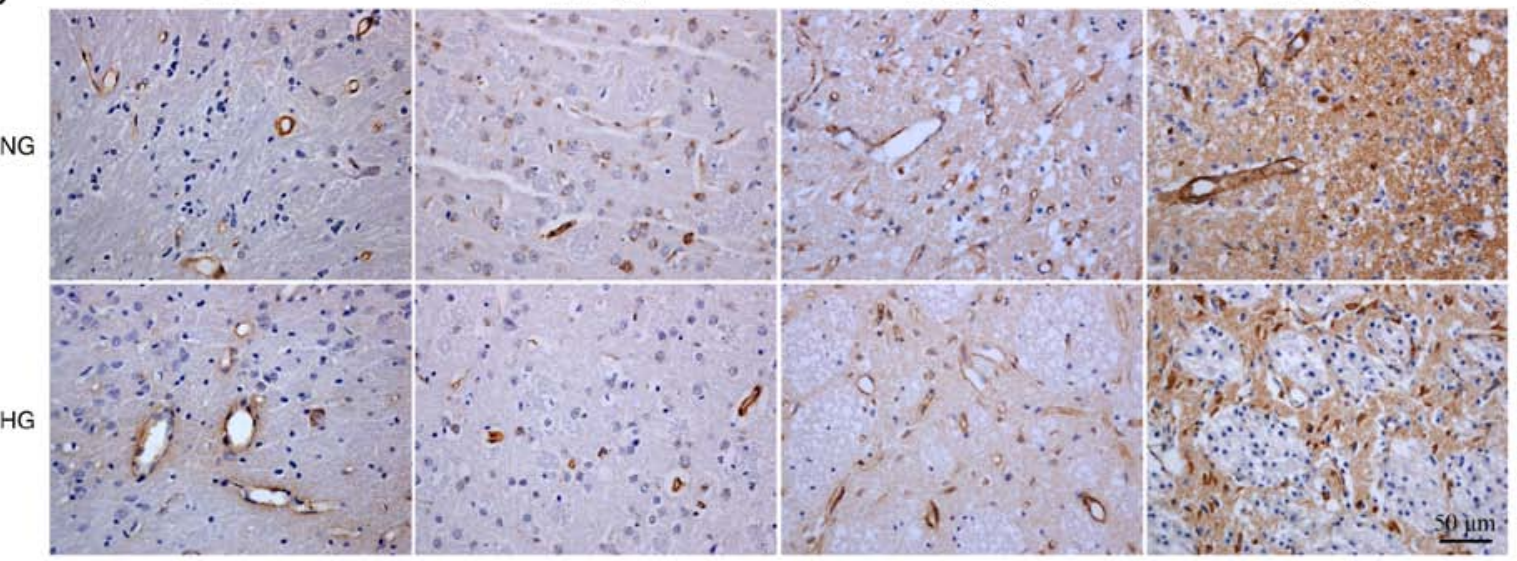

C

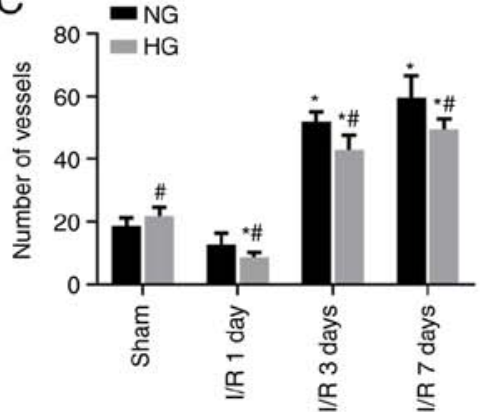

D

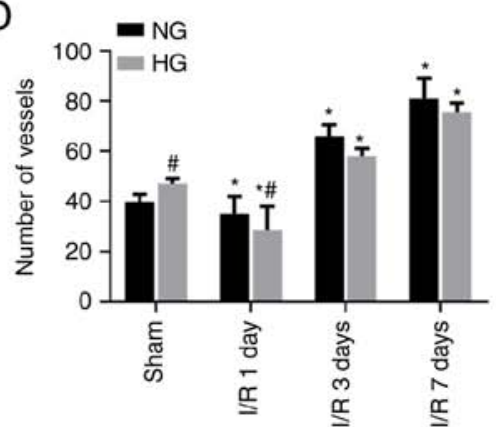

$\mathrm{E}$

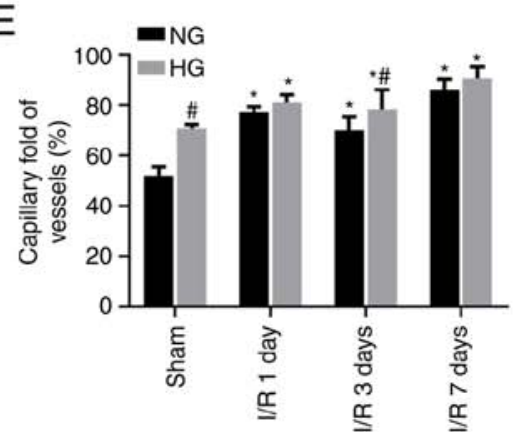

Figure 3. Changes of vascular density following I/R Immunohistochemistry of von Willebrand factor in the ischemic penumbra of (A) cortex and (B) striatum. Number of cerebral vessels in (C) cortex and (D) striatum. (E) Quantitative summary of the ratio of capillaries ( $<10 \mu \mathrm{m}$ diameter) to all cerebral vessels/brain section. $\mathrm{n}=5$ /group. ${ }^{*} \mathrm{P}<0.05$ vs. Sham, ${ }^{\text {}} \mathrm{P}<0.05$ vs. NG. NG, normoglycemic; HG, hyperglycemic; I/R, ischemia/reperfusion.

glucose levels of $\mathrm{HG}$ animals elevated to $>16.7 \mathrm{mM}$ at 1 week post-STZ injection and further increased to $22-34 \mathrm{mM}$ in 2-4 weeks post-STZ injection. By contrast, the blood glucose in NG animals stayed at baseline levels of $5-8 \mathrm{mM}(\mathrm{P}<0.01 \mathrm{vs}$. HG; Fig. 1C). Neurological function was assessed using the mNSS at 1 day post-reperfusion (Fig. 1D). The sham control animals had a mean score of 2. Cerebral ischemia in NG animals increased the deficit score to 9 ( $\mathrm{P}<0.01 \mathrm{vs}$. Sham) and to 13.6 in $\mathrm{HG}$ ischemic animals $(\mathrm{P}<0.05$ vs. $\mathrm{NG})$.

Hyperglycemia aggravates brain injury and increases permeability of the BBB following I/R. TTC \& Evans blue double staining were used to evaluate the infarct volume and BBB disruption. The normal non-ischemic brain tissue was stained pink while the ischemic infarcted area remained white. When the permeability of the BBB increased, Evans blue penetrated the $\mathrm{BBB}$ via blood vessels, staining brain tissue blue. TTC staining revealed that ischemia in NG animals resulted in $29 \%$ infarct volume at 1 day recovery. As expected, preischemic hyperglycemia enlarged the infarct volume to $45 \%$ (Fig. 2A and B). The area of Evans blue staining in $\mathrm{NG}$ animals was $11 \%$ leaking area; in $\mathrm{HG}$ animals, this was increased to $19 \%(\mathrm{P}<0.05$ vs. $\mathrm{NG})$, suggesting $\mathrm{HG}$ exacerbated $\mathrm{BBB}$ leakage at 1 day reperfusion (Fig. 2A and $\mathrm{C}$ ). In order to confirm that $\mathrm{HG}$ increased the permeability of the BBB following I/R injury, IgG staining was performed (Fig. 2D and E). The results demonstrated that ischemia in NG animals led to moderately increased $\operatorname{IgG}$ staining in 
A

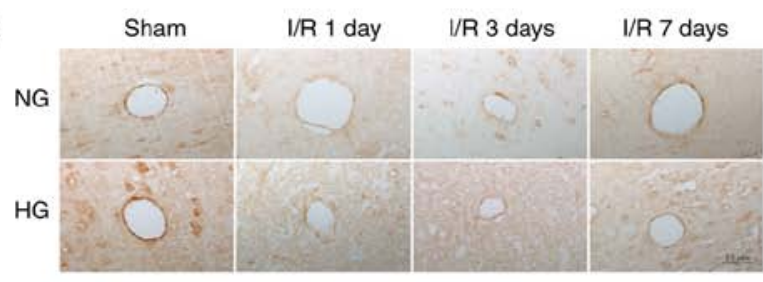

B

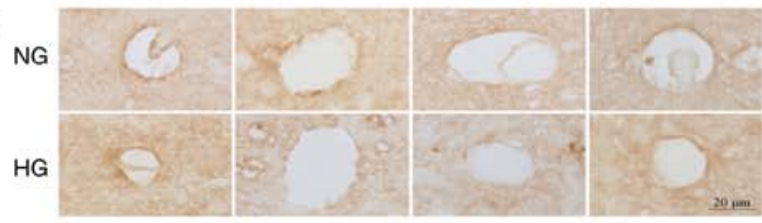

C

NG

$H G$
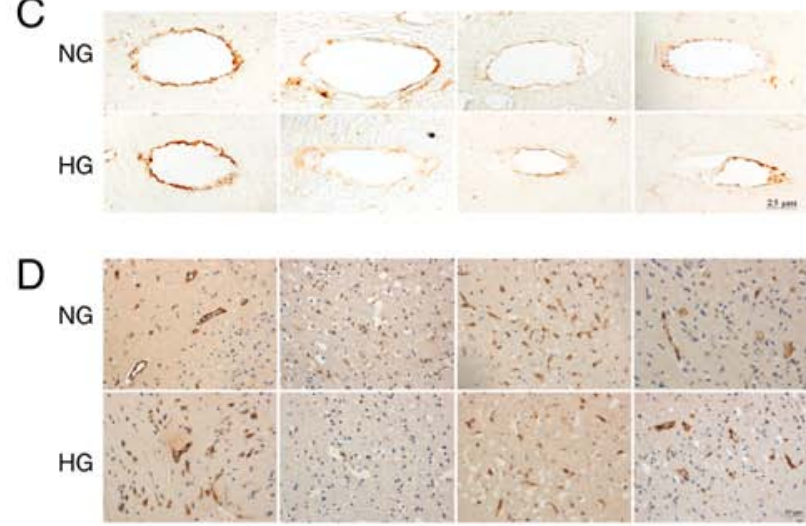

$\mathrm{E}$

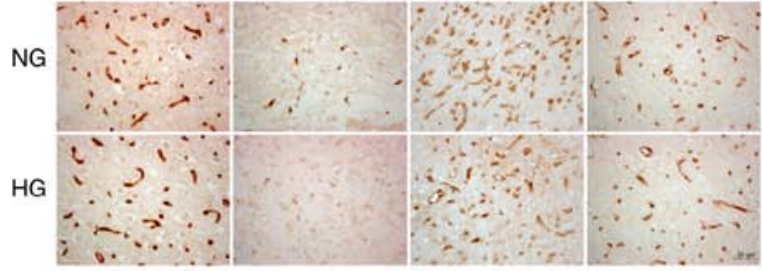

F
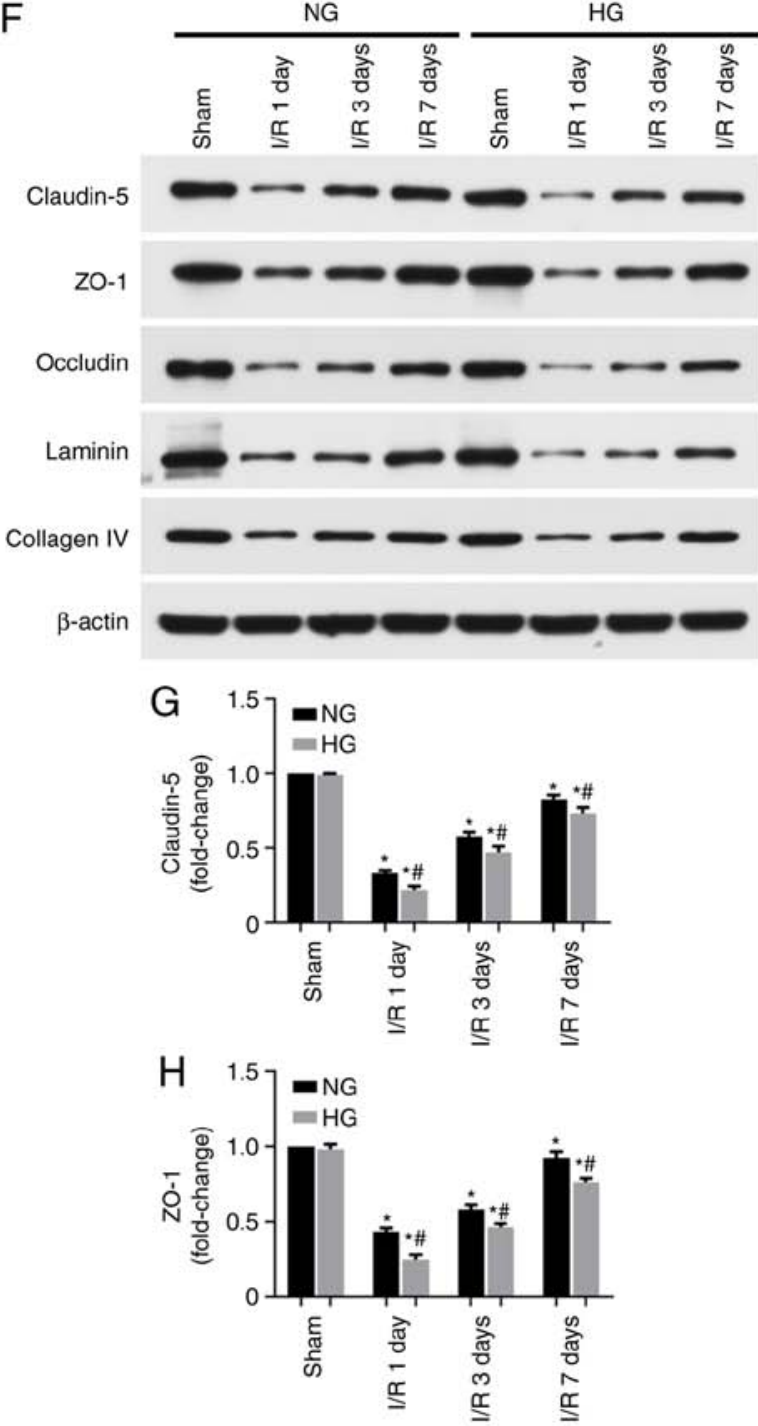
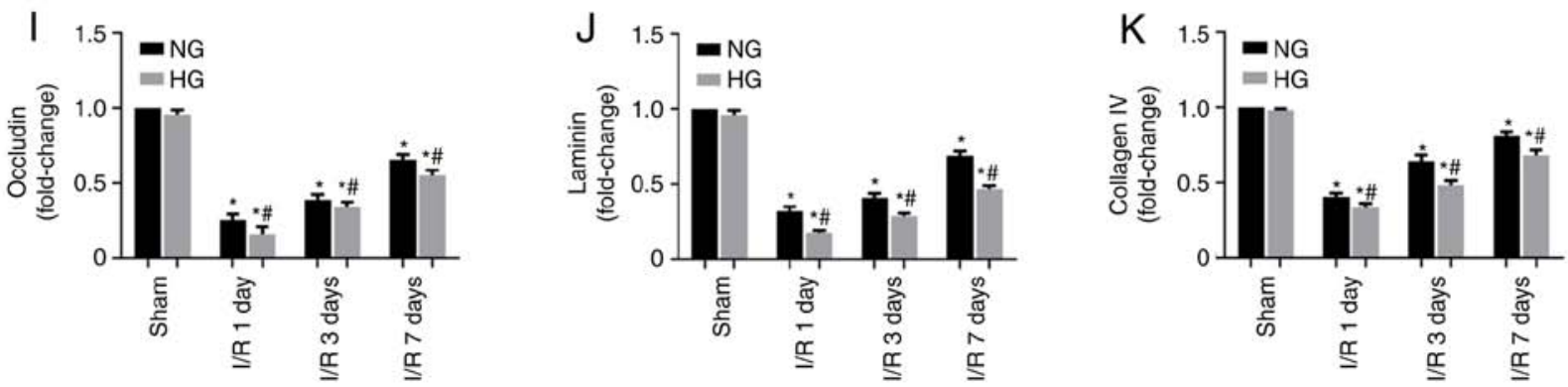

Figure 4. Immunoreactivity and levels of tight junction and basement membrane proteins following cerebral I/R injury. Representative photomicrographs showing (A) claudin-5, (B) ZO-1, (C) occludin, (D) laminin and (E) collagen IV staining in the ischemic cortical penumbra (n=5/group). (F) Representative western blots and relative amounts of (G) claudin-5, (H) ZO-1, (I) occludin, (J) laminin and (K) collagen IV (n=6/group). ${ }^{*} \mathrm{P}<0.05$ vs. Sham, "P $\mathrm{P}<0.05$ vs. NG. $\mathrm{I} / \mathrm{R}$, ischemia/reperfusion; ZO-1, zonula occluden-1; NG, normoglycemic; HG, hyperglycemic.

the right middle cerebral artery territory. In HG animals, the IgG-stained area was significantly increased $(\mathrm{P}<0.01 \mathrm{vs}$. $\mathrm{NG}$ ), confirming that hyperglycemia damaged the integrity of the BBB. Evaluation of brain edema by dry-wet weight ratio revealed that brain edema was mildly and moderately increased in NG and HG animals, respectively.

The effect of HG on histopathology of brain tissue at the frontal cortex was evaluated using hematoxylin and eosin (H\&E) staining. Sham group exhibited normal brain structure with no evidence of tissue swelling, necrosis or other visible abnormalities (Fig. 2G). No abnormalities were observed in HG Sham animals, aside from an increased number of microvessels compared with the NG Sham group. At 1 day post-I/R, cerebral infarction, which is characterized by brain edema, loosening of the matrix, pyknotic neurons and loss of blood vessels (42), was observed. These changes were significantly worsened in the HG group compared with the NG group. At 3- and 7-days post-reperfusion, glial cell proliferation and irregular neovascularization appeared. Glial cell infiltration in $\mathrm{HG}$ animals was less than that in the NG group. 


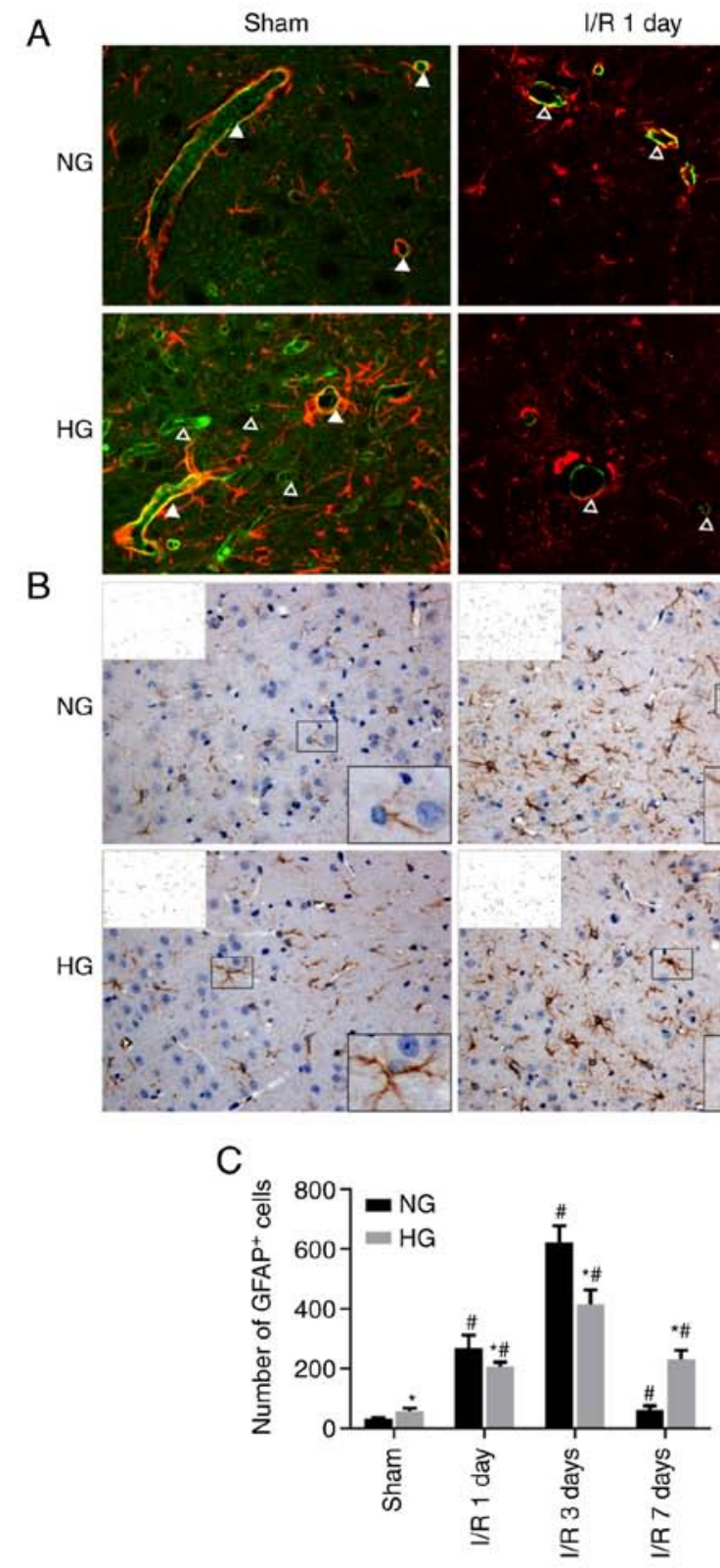

E

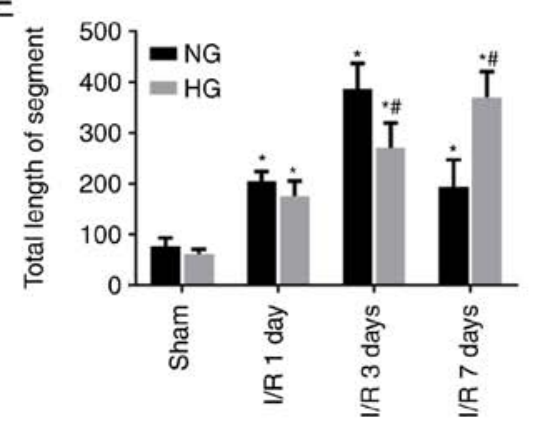

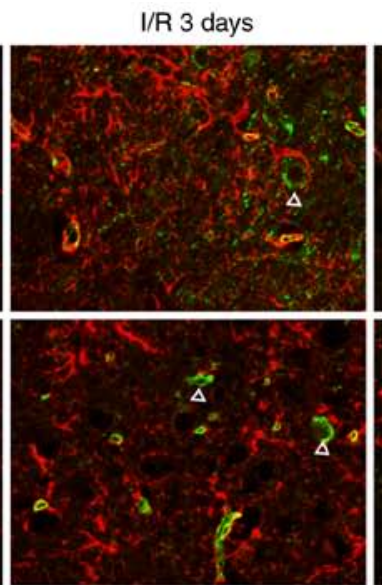
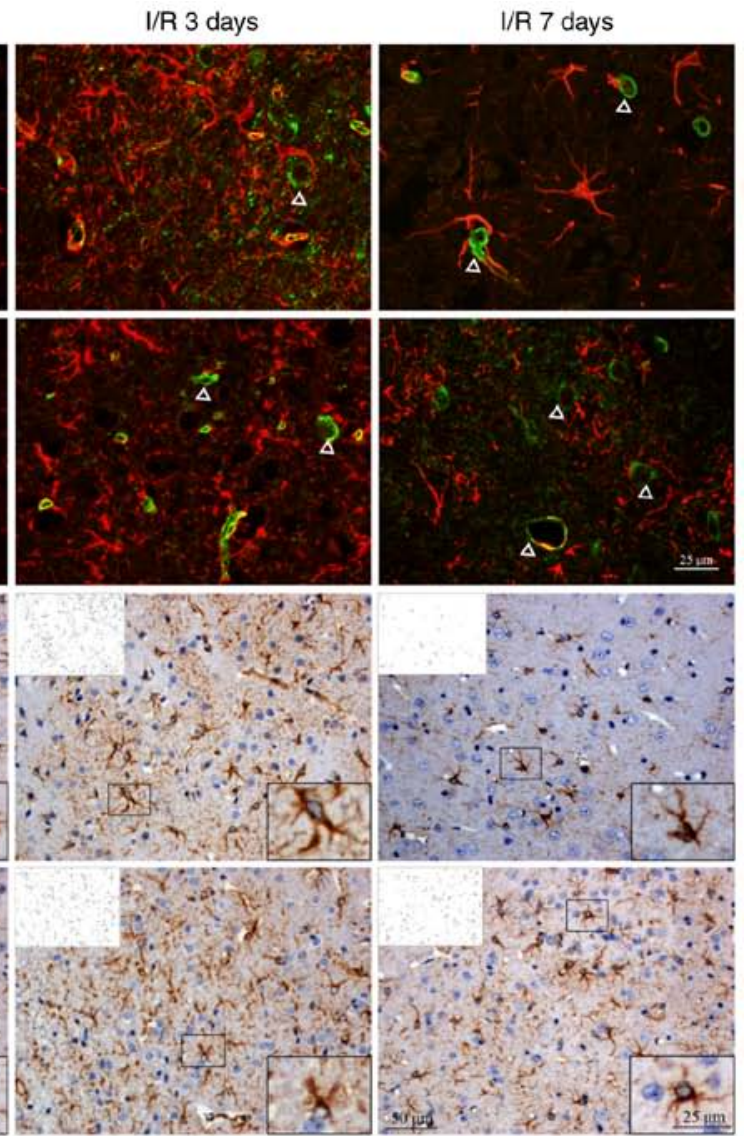

D

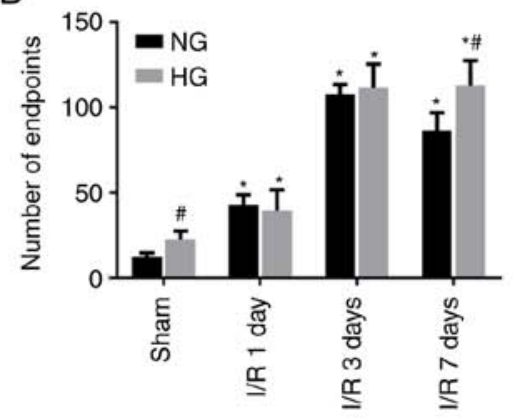

$\mathrm{F}$

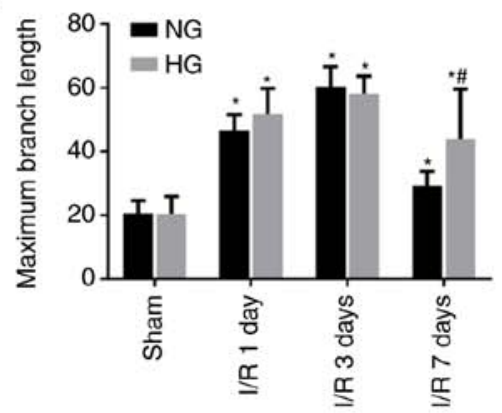

Figure 5. Changes of endfeet following I/R. (A) Immunofluorescent double labeling of von Willebrand factor (green) and GFAP (red) in the ischemic cortical penumbra. White solid triangles indicate vessels completely surrounded by astrocytic endfeet; hollow triangles indicate vessels with incomplete/absent astrocytic endfeet. (B) GFAP immunohistochemistry in the ischemic cortical penumbra. Magnified skeletonized insets (top left) and enlarged inserts (bottom right) demonstrate morphological changes of astrocytes at different times post-I/R injury. Quantitative summary of (C) GFAP-positive astrocytes, (D) endpoints, (E) total segment length and (F) maximum branch length. $n=5 /$ group. ${ }^{*} \mathrm{P}<0.05$ vs. Sham, ${ }^{\text {}} \mathrm{P}<0.05$ vs. NG. GFAP, glial fibrillary acidic protein; NG, normoglycemic; HG, hyperglycemic; I/R, ischemia/reperfusion.

Effect of hyperglycemia on cerebral vessel density following cerebral I/R injury. In order to observe the influence of HG ischemia on cerebral blood vessels, vascular density was evaluated by labeling vascular endothelial cells via vWF staining and counting the number of vessels in NG- and HG-ischemic rats (Fig. 3). Consistent with the observations in 

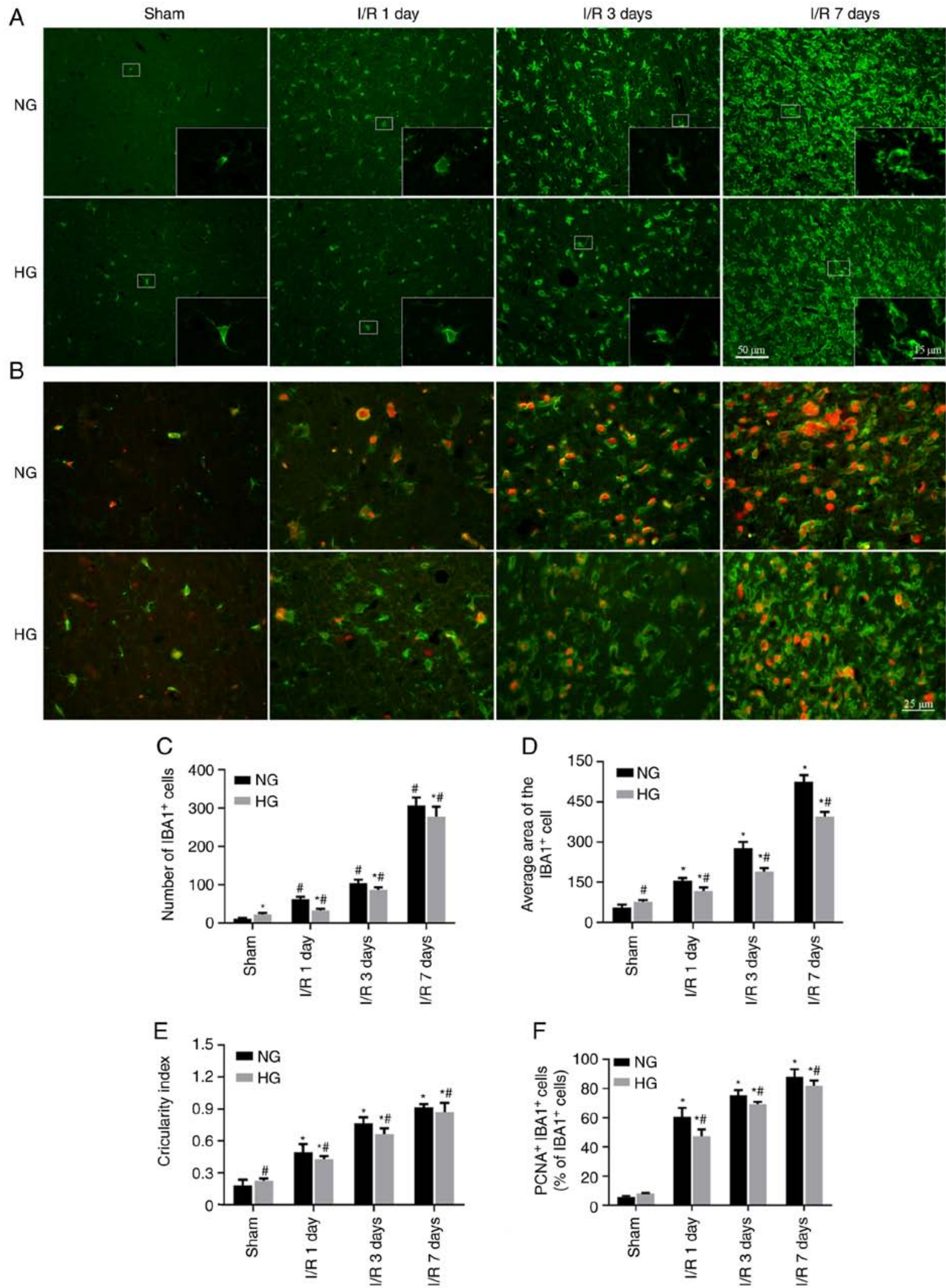

Figure 6. Immunofluorescent double labelling of microglial marker IBA1 (green) and proliferation marker PCNA (red). (A) Representative photomicrographs showing IBA1 staining in the ischemic cortex penumbra. Enlarged inserts indicate morphology of activated microglia. (B) Co-localization of IBA1 and PCNA in the ischemic cortical penumbra. (C) Number and (D) average area of IBA1-stained microglial cells. (E) Circularity index. (F) Percent of PCNA ${ }^{+} / \mathrm{IBA1}^{+}$cells to all $\mathrm{IBA}^{+}$cells indicates microglial proliferation. $\mathrm{n}=5$ /group. ${ }^{*} \mathrm{P}<0.05$ vs. Sham, ${ }^{*} \mathrm{P}<0.05$ vs. NG. IBA1, ionized calcium-binding adaptor molecule; PCNA, proliferating cell nuclear antigen; NG, normoglycemic; HG, hyperglycemic; I/R, ischemia/reperfusion.

H\&E-stained sections, the number of vessels in both cortex and striatum regions significantly increased in the HG Sham group compared with the NG Sham group. The number of blood vessels initially decreased after 1 day and significantly 


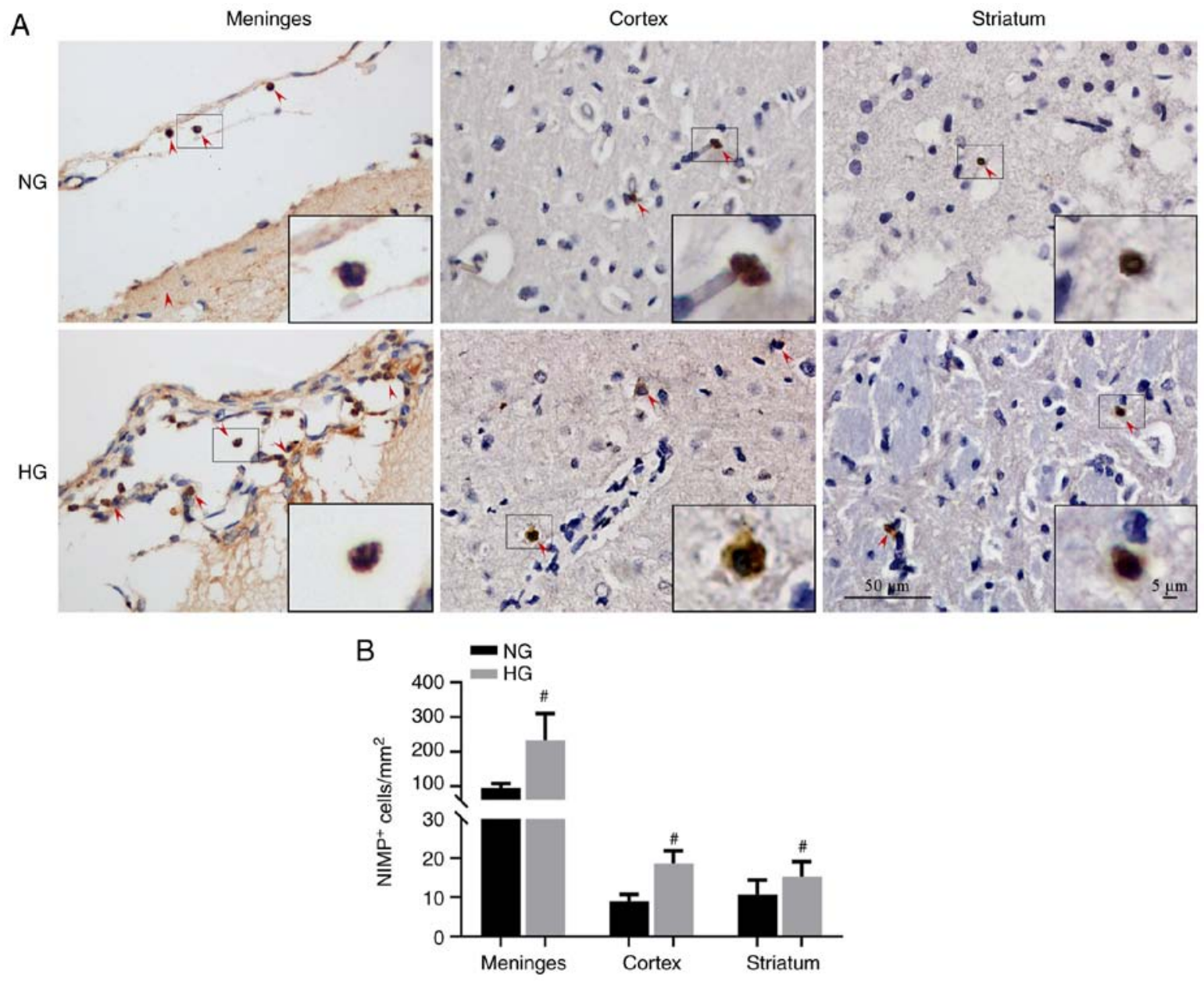

Figure 7. Neutrophil infiltration following I/R (A) NIMP-R14 staining showing neutrophil infiltration in the meninges and brain parenchyma (cortex and striatum) following cerebral I/R injury. Arrows indicate NIMP-R14+ neutrophils; enlarged insets demonstrate polymorphonuclear features of neutrophil. (B) Neutrophil density in different areas of the brain. $\mathrm{n}=5 /$ group. ${ }^{*} \mathrm{P}<0.05$ vs. NG. I/R, ischemia/reperfusion; NG, normoglycemic; HG, hyperglycemic; NIMP-R14, anti-neutrophil antibody.

increased in both structures at 3 and 7 days post-I/R in NG animals. Similarly, the number of blood vessels decreased at 1 day and significantly increased in the cortex and striatum at 3 and 7 days post-I/R in $\mathrm{HG}$ animals, but the extent of these increases were less than those in NG animals (Fig. 3A-D).

Blood vessels with diameters $<10 \mu \mathrm{m}$ were defined as capillaries and the ratio of capillaries to all vessels was calculated. The ratio in the NG Sham group was $47.6 \%$; in the HG Sham group, this was increased to $70.2 \%$ (Fig. 3E). Following I/R injury, the capillary ratio increased significantly at 1-7 days post-reperfusion compared with sham groups but there was no significant difference between the NG and HG groups.

$T J$ and $B M$ are damaged by hyperglycemia following $I / R$. TJs are the most important barrier structure of the BBB. They are primarily composed of claudins, occludins and ZO-1 (43). These three proteins were distributed continuously and uniformly among vascular endothelial cells with a granular shape in NG and HG Sham groups. At 1 day post-reperfusion, TJ proteins were distributed intermittently among vascular endothelial cells with loss of continuity and decreased staining intensity. These changes were more notable in the HG group than in the NG group. At 3 and 7 days post-reperfusion, TJ proteins recovered continuity of distribution around the vessels and increased intensity of staining; this recovery was slower in HG compared with NG animals (Fig. 4A-C).

Laminin and collagen IV constitute the network skeleton of the BM (44). In the Sham NG and HG groups, laminin and collagen IV were distributed in the vessels and their shapes could be clearly seen (dark brown). At 1 day post-reperfusion, the two proteins were lost along with infarct formation in brain tissue of both NG and HG animals, with more pronounced changes observed in the HG animals. At 3 and 7 days post-reperfusion, immunoreactivity of the BM protein was detected, along with the neovascularization of cerebral vessels. The density of laminin and collagen IV was weaker in HG compared with NG groups (Fig. 4D and E).

The aforementioned observations were confirmed by western blot analysis of the proteins. Similar to the observations in IHC, the levels of these proteins decreased sharply at 1 day post-reperfusion and increased gradually from 3 to 7 days post-reperfusion. Compared with NG animals, the levels of all five aforementioned proteins were lower in $\mathrm{HG}$ animals (Fig. 4F-K).

Damage to astrocytic endfeet and astrocytic activation. The astrocytic endfoot is an important component of the BBB (45). In the Sham HG and NG groups, vWF-labeled vessels were 
A

\begin{tabular}{|c|c|c|c|c|c|c|c|}
\hline \multicolumn{4}{|c|}{$N G$} & \multicolumn{4}{|c|}{$H G$} \\
\hline 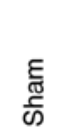 & 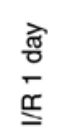 & $\begin{array}{l}\text { D. } \\
\text { శ్ } \\
0 \\
\cong \\
\subseteq\end{array}$ & 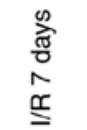 & 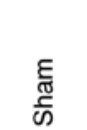 & 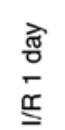 & 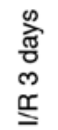 & 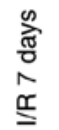 \\
\hline- & $=$ & - & $\longrightarrow$ & - & & & \\
\hline
\end{tabular}

IL-1 $1 \beta$

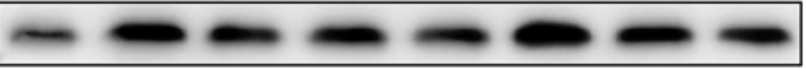

IL-6

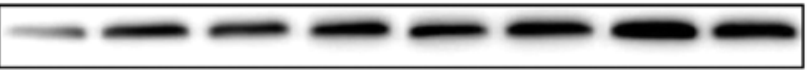

$\beta$-actin

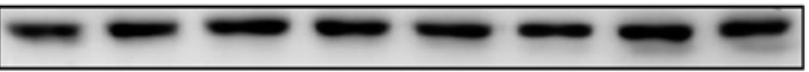

B
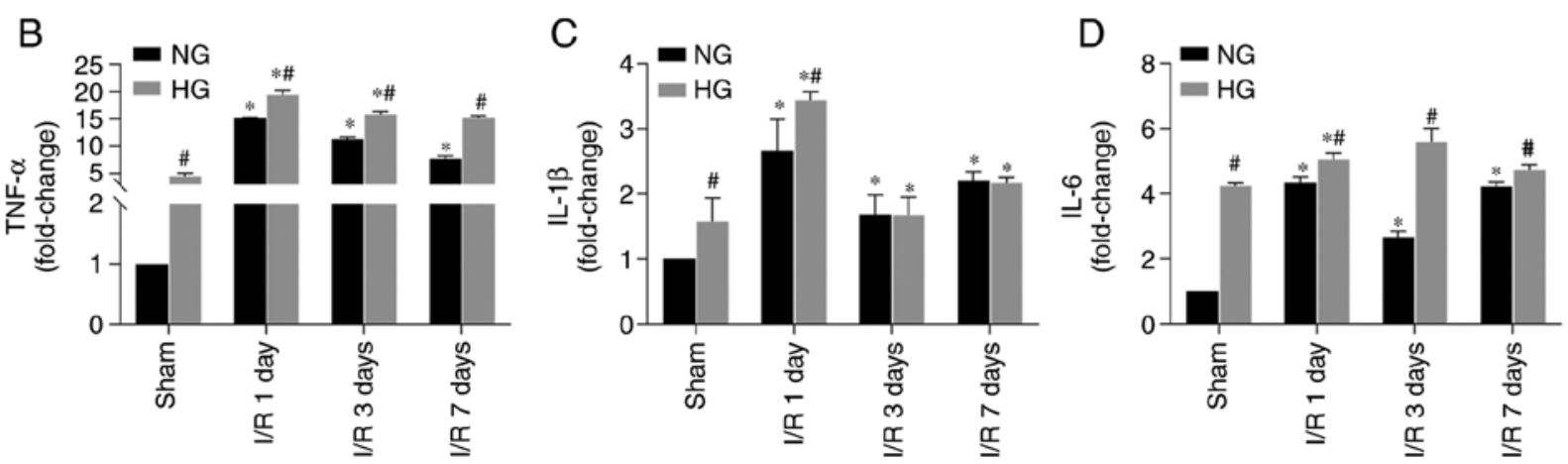

Figure 8. Detection of inflammatory cytokines. (A) Western blots and ratio of (B) TNF- $\alpha,(\mathrm{C})$ IL-1 $\beta$ and (D) IL-6 compared with $\beta$-actin (n=6). *P<0.05 vs. Sham, ${ }^{\#} \mathrm{P}<0.05$ vs. NG. NG, normoglycemic; HG, hyperglycemic; I/R, ischemia/reperfusion.

tightly surrounded by GFAP-stained astrocyte endfeet. This was observed in both large and small vessels in the NG Sham group. At 1 day post-reperfusion, most of the vessels lost astrocytic endfeet and only a few large vessels were partially surrounded by astrocytes in the NG and HG groups. By contrast, HG Sham group exhibited more blood vessels than the NG Sham group. At 1 day recovery, both large and small vessels lost astrocyte endfoot wrappings in $\mathrm{HG}$ animals (Fig. 5A).

IHC of astrocytes using anti-GFAP antibody demonstrated that astrocytes had small cell bodies and fewer dendrites in NG Sham group compared with the HG Sham group. Number of astrocytes increased moderately after 1 day, peaked at 3 days and slightly declined at 7 days in NG animals. Compared with NG Sham, the astrocyte bodies enlarged and exhibited increased number of dendritic endpoints, length of the dendrite segment and maximum branch length of dendrites in NG I/R animals (Fig. 5B-F), suggesting that astrocytes were activated by $\mathrm{I} / \mathrm{R}$ in NG animals. Compared with NG animals, the number of astrocytes increased slightly in the HG Sham group. Following I/R, the number of astrocytes in NG groups increased to lesser extent at days 1 and 3 but was higher at day 7 than those in $\mathrm{HG} \mathrm{I/R}$ at corresponding endpoints. The number of endpoints, total length of branch segments and maximum branch length increased in $\mathrm{HG} 1$ and 3 day groups but did not exceed those in NG animals, but exhibited higher values at 7 days compared with NG (Fig. 5B-F). These data suggest that HG activated astrocytes and HG-activated astrocytes persisted longer than in NG animals following I/R.

Inhibition of microglia by hyperglycemia following cerebral $I / R$ injury. As innate immune cells in the CNS, microglia remove harmful substances that enter the brain parenchyma (41). Microglial activation was assessed by labeling IBA1 [a marker of mature microglia (46)] coupled with morphological analysis. The results (Fig. 6) showed that in the NG Sham group, microglia remained in a survey state, characterized by a ramified morphology with long branches and small cellular body. However, in the HG Sham group, the number of microglia cells increased and amoeboid morphology with a larger cellular body and shorter branches were observed, indicating activation of microglial cells by HG. Following I/R injury, the number of IBA1-stained cells increased from days 1 to 7 in NG animals, as did the average area of the microglial cells, circularity index and newly generated microglial cells, reflected by positive double labelling of IBA1 with cell proliferation marker PCNA (Fig. 6A-F). The activated microglia exhibited typical morphological characteristics, such as thickening and retraction of dendrites and enlargement of cell body to nearly circular shape (Fig. 6E). Compared with NG ischemic animals, the number and average area of microglial cells, as well as the number of newly generated microglial cells, decreased in the corresponding HG group, suggesting that the activation of microglia in $\mathrm{HG} \mathrm{I/R}$ group was as strong as in the NG I/R counterparts (Fig. 6A-F).

Neutrophil infiltration increases in the meninges of $H G I / R$ animals. Neutrophil infiltration damages the BBB, which further increases neutrophil infiltration (47). Neutrophil infiltration was investigated by labeling brain sections with neutrophil antibody (anti-NIMP-R14) followed by IHC analysis of meningeal and brain parenchyma (cortex and striatum) with respect to the number of NIMP-R14+ ${ }^{+}$neutrophils. NG 


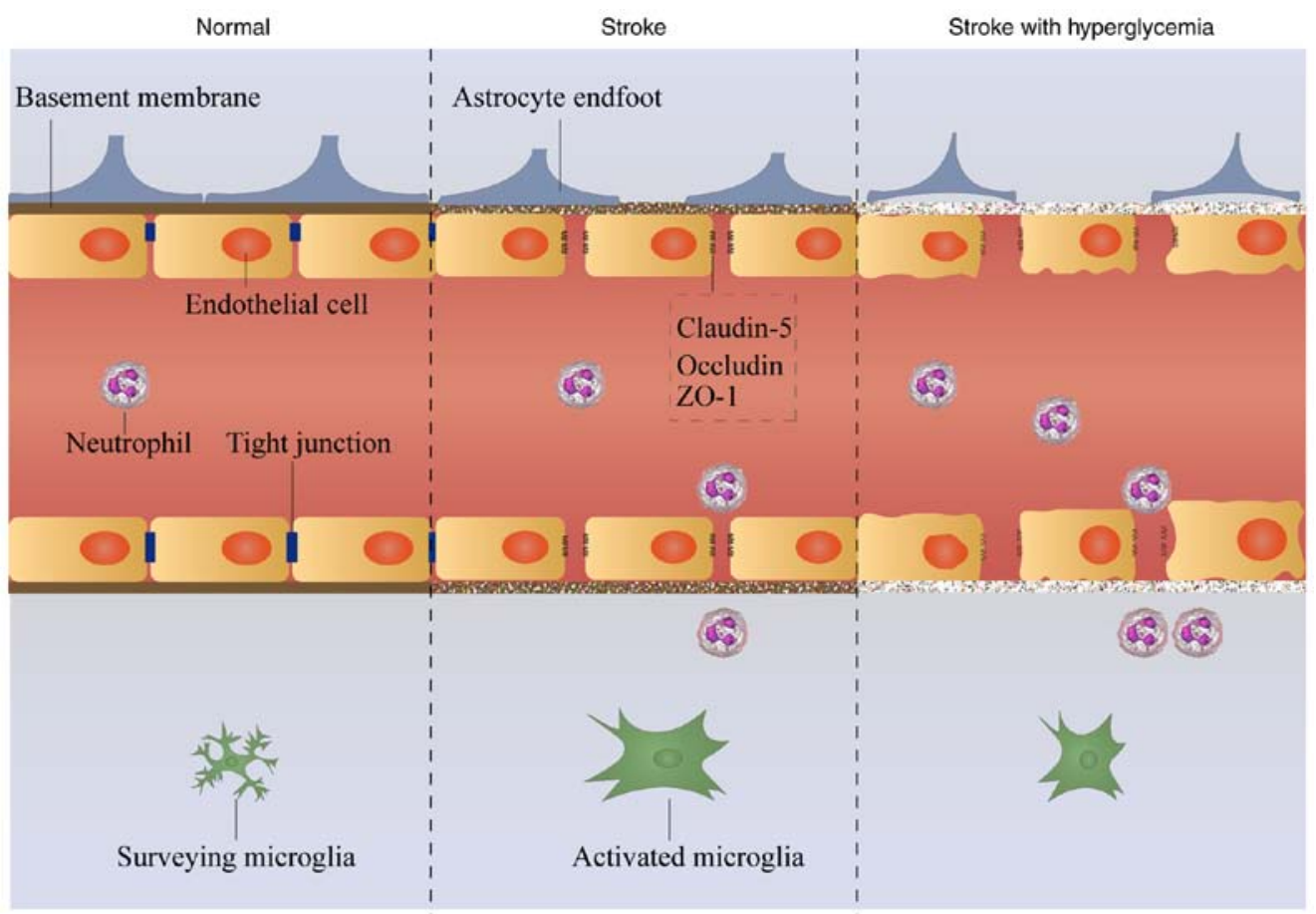

Figure 9. Schematic diagram of structural changes of the BBB following middle cerebral artery occlusion in NG and HG animals. Ischemia/reperfusion causes degradation of tight junctions and basement membrane and loss of astrocytic endfeet. Hyperglycemia further aggravates the damage and results in increased BBB permeability compared with NG animals. HG suppresses activation and proliferation of microglia and increased neutrophil infiltration, which may contribute to its detrimental effect on ischemic brain damage. BBB, blood-brain barrier; NG, normoglycemic; HG, hyperglycemic; ZO-1, zonula occluden-1.

I/R-increased neutrophil infiltration primarily occurred in the meninges and $\mathrm{HG}$ I/R caused further increases. In the brain parenchyma, only a small number of NIMP-R $14^{+}$cells were detected and there was no significant difference between the NG I/R and HG I/R groups (Fig. 7A and B).

Inflammation increases in the penumbra of $H G I / R$ animals. In order to determine the change in neuroinflammation, protein levels of pro-inflammatory factors TNF- $\alpha$, IL-1 $\beta$ and IL- 6 in the cortical penumbral tissue were measured. Protein levels of these three factors were significantly increased following I/R in NG animals, with peak levels at 1 day post-I/R (Fig. 8). Compared with NG I/R animals, the increases were further enhanced in the HG I/R groups. In addition, animals in the HG sham groups also exhibited increased levels of inflammatory markers, suggesting hyperglycemia-alone caused stress to brain. This was consistent with observations that astrocytes and microglia were activated in the HG Sham group.

\section{Discussion}

Both animal and clinical studies have confirmed that diabetes is an important independent risk factor for stroke (48-51). Compared with non-diabetic patients, people with diabetes are more likely to suffer stroke (52). Furthermore, the size of cerebral infarction, mortality and rate of disability are significantly increased following stroke in patients with hyperglycemia or diabetes $(53,54)$. In the present study, HG animals exhibited more severe cerebral edema, larger infarct volume and higher neurological deficit scores than NG animals following $30 \mathrm{~min}$ ischemia and $24 \mathrm{~h}$ reperfusion. The pathomorphological results showed that hyperglycemia aggravated brain tissue damage; more severe pan-necrosis was observed in the brain sections from $\mathrm{HG}$ compared with those from from NG animals at days 1, 3 and 7 post-reperfusion. These results support the hypothesis that hyperglycemia is an aggravating factor in cerebral I/R injury. In addition, hyperglycemia-alone increased the number of small vessels and capillaries, as observed by routine H\&E and vascular specific vWF staining. The percentage of capillaries increased in HG Sham animals, suggesting hyperglycemia may increase vasogenesis in normal healthy animals (55).

Over the past few decades, a number of clinical trials have examined the efficacy of neuroprotective agents $(56,57)$, however, none of them improved clinical recovery in patients following a stroke (58). Lo et al (10) proposed the concept of the NVU in 2003, which refers to an overall structural and functional unit consisting of neurons, endothelial cells, glial cells with pericytes, BM and extracellular matrix $(10,59,60)$. As a core structure of the NVU, the BBB not only prevents harmful substances entering the brain parenchyma but also regulates endothelial-glial and glial cell-neuron interaction. In order to determine the effects of hyperglycemia on the BBB following $\mathrm{I} / \mathrm{R}$, the integrity of the $\mathrm{BBB}$ was assessed using Evans blue and IgG leakage assays. HG I/R significantly increased leakage of both Evans blue and $\mathrm{IgG}$ from cerebral blood vessels to the brain parenchyma, suggesting that hyperglycemia damaged the BBB and increased its permeability. This finding is consistent with results reported by Kamada et al (30), who demonstrated that MMP-9 is activated following HG I/R injury.

TJs, primarily composed of claudin-5, occludin and ZO-1 proteins, are key for maintaining structure and function of the BBB (33). Claudin-5 is a TJ-specific protein necessary for TJ 
formation and BBB function (61). Occludin is a cell polarity protein that primarily has the function of palisade and barrier regulation. The palisade function is to divide the plasma membrane of epithelial cells into functional zones (the lipid part of the top and the protein part of the basal side) while preventing mutual diffusion between the functional areas, which mediates formation of cell polarity. The barrier regulation function is selective to transmembrane materials (15,62-64). It regulates the transport of small molecules between cells and maintains brain tissue homeostasis (65). $\mathrm{ZO}-1$ protein is key to TJ stability and function (66-68). Dissociation of ZO-1 from the TJ complex is associated with increased permeability of the BBB (69). Since these three proteins are the major structural proteins of the TJ, changes in their expression result in alteration of BBB permeability and brain edema $(70,71)$. In the present study, hyperglycemia notably suppressed protein levels and exacerbated discontinuity of these TJ proteins around vessels following I/R injury compared with $\mathrm{NG}$ animals, suggesting that hyperglycemia-induced BBB leakage resulted from damage to TJ proteins.

The BM is the second barrier structure of the BBB. Located at the interface of the circulation system and the $\mathrm{CNS}$, the $\mathrm{BM}$ is well positioned to regulate BBB integrity under both homeostatic and pathological conditions. The brain BM primarily consists of laminin, collagen IV, nidogen and heparan sulfate proteoglycans. Laminin and collagen IV are the two most abundant components, which constitute the basic framework of the BM. Cerebral ischemia damages BM components, including laminin and collagen IV in gerbil, rat and primate models of cerebral I/R injury, potentially due to activation of MMP-9 (20,22,44,72-74). In the present study, hyperglycemia further decreased the levels of laminin and collagen IV at 1, 3 and 7 days post-reperfusion compared with NG animals at identical time points, suggesting that $\mathrm{HG}$ ischemia causes more severe damage to the BM than NG ischemia. Suppression of TJ proteins and damage to the BM by hyperglycemia may explain why there is an increased transition from ischemic to hemorrhagic stroke in animal stroke models and clinical patients with stroke $(75,76)$.

Astrocytes are the most abundant cell type in the CNS (77). They support and protect neurons by maintaining the homeostatic balance of the neural microenvironment (78). Astrocytes also interact with endothelial cells and support BBB integrity via endfeet that encircle cerebral blood vessels (27). In our previous study, partial or complete absence of astrocyte endfeet around the blood vessel wall was observed in diabetic animals following MCAO (79). In the present study, the loss of astrocytic endfeet following I/R injury was primarily observed in the small cerebral blood vessels in the NG group, while hyperglycemia exacerbated loss of endfeet to all types of cerebral blood vessel. This variable damaging effect of HG and NG on endfeet of blood vessels with different diameters may be one of the mechanisms underlying the exacerbated damage induced by hyperglycemia on the BBB. Several studies have shown that activated astrocytes release trophic factors and extracellular matrix molecules, which promote neuronal survival and plasticity following ischemia (80-82).

In the present study, astrocytes were activated at day 1 post-reperfusion following ischemia. In NG animals, the activation peaked at 3 days post-I/R, and then tapered off at 7 days. Changes of astrocytes, such as activation, in $\mathrm{HG}$ animals followed the same trend as seen in NG groups. However, the number of $\mathrm{GFAP}^{+}$cells and endpoints, total length of dendrite segments and maximum branch length were significantly higher at 7 days in HG compared with NG animals. These results suggest that hyperglycemia prolonged the duration of astrocytic activation. This may facilitate tissue repair; however, continuously activated astrocytes form glial scar tissue around the necrotic brain tissue of the infarct, which provides a physical barrier against axonal growth, thus preventing neurological recovery following stroke $(60,83)$.

Activation of microglial cells occurs in CNS infectious disease and neurodegenerative disorders. The activation of microglia has also been shown to regulate brain endothelial cell proliferation (84). The present results showed that cerebral $\mathrm{I} / \mathrm{R}$ injury resulted in a time-dependent activation of microglial cells in the brain, as reflected by increases in number of IBA- $1^{+}$ cells, average area of the microglia and circularity index. I/R increased microglial cell proliferation, as detected by double positive labeling of IBA-1 and PCNA. Compared with NG animals, hyperglycemia repressed activation and proliferation of microglial cells. This may suppress the immune response and tissue repair in the reperfusion stage.

The infiltration of neutrophils serves an important role in mediating post-stroke damage in the brain. It causes increased production of free radicals, exacerbation of neuroinflammation and disruption of the $\mathrm{BBB}$ and hemorrhagic transformation post-stroke (35). Activation of neutrophils via lipopolysaccharide notably enhances BBB disruption in an animal model of stroke (85). By contrast, inhibition of neutrophils alleviates BBB leakage following stroke and decreases the risk of hemorrhagic transformation following thrombolysis (86). Consistent with these reports, the present study demonstrated $\sim 3$-fold increases in numbers of of neutrophils in the meninges of $\mathrm{HG}$ animals compared with the NG group; no significant difference in the number of neutrophils in the brain parenchyma or striatum was observed between groups.

Activation of microglia and infiltration of neutrophils may elicit release of pro-inflammatory cytokines, which contribute to neuronal damage following stroke (87). Increases in levels of TNF- $\alpha$, IL- $1 \beta$ and IL- 6 were observed at 1 day post-I/R; these levels decreased at 3 and 7 days post-I/R in NG animals. The changes in expression levels of these inflammatory cytokines followed the same trend in HG groups; however, the levels were higher than in the corresponding NG groups. These results are consistent with the increased infiltration of neutrophils in HG groups, suggesting that hyperglycemia aggravated the ischemia-induced neuroinflammatory response.

In conclusion, hyperglycemia increased BBB leakage, suppressed levels of TJ and BM proteins and damaged astrocyte endfeet that enclose cerebral vasculature following cerebral I/R injury. Furthermore, hyperglycemia suppressed microglial activation and proliferation, enhanced neutrophil infiltration into the brain and increased levels of inflammatory cytokines. It was hypothesized that hyperglycemia-exacerbated BBB damage is caused by damage to TJs, BM and astrocytes, and is associated with increased neuroinflammation (Fig. 9). The present study aimed to determine the underlying mechanism of aggravated BBB damage in diabetes. However, other aspects, such as the association or cascade reaction between 
these structures (endothelial cells, TJs, astrocytes, BM and microglia) and the role of pericytes, an important barrier for the BBB $(66,67)$, deserve further investigation.

\section{Acknowledgements}

The authors would like to thank Mrs Laretha Davis (North Carolina Central University, NC, USA) for editing and proofreading.

\section{Funding}

The present study was supported by the National Natural Science Foundation of China (grant nos. 31960177 and 31780280) and the Natural Science Foundation of Ningxia (grant no. 2018AAC03092).

\section{Availability of data and materials}

The datasets used and analyzed in the current study are available from the corresponding authors on reasonable request.

\section{Authors' contributions}

YZG conceived the study. YZG and JZZ performed data analysis. LJ and JZZ acquired funding. LDD and AG performed the experiments. JZZ was responsible for project administration. JWZ established the experimental animal model. LJ supervised the study and performed the MCAO model. TD and PAAL analyzed data and wrote the original draft of the manuscript. JZZ and YZG confirm the authenticity of all the raw data. All authors read and approved the final manuscript.

\section{Ethics approval and consent to participate}

The present study was approved by the Institutional Animal Care and Use Committee of Ningxia Medical University.

\section{Patient consent for publication}

Not applicable.

\section{Competing interests}

The authors declare that they have no competing interests.

\section{References}

1. Li W, Prakash R, Kelly-Cobbs AI, Ogbi S, Kozak A, El-Remessy AB, Schreihofer DA, Fagan SC and Ergul A: Adaptive cerebral neovascularization in a model of type 2 diabetes: Relevance to focal cerebral ischemia. Diabetes 59: 228-235, 2010.

2. Chen R, Ovbiagele B and Feng W: Diabetes and stroke: Epidemiology, pathophysiology, pharmaceuticals and outcomes Am J Med Sci 351: 380-386, 2016.

3. Li W, Qu Z, Prakash R, Chung C, Ma H, Hoda MN, Fagan SC and Ergul A: Comparative analysis of the neurovascular injury and functional outcomes in experimental stroke models in diabetic Goto-Kakizaki rats. Brain Res 1541: 106-114, 2013.

4. Hu Q, Manaenko A, Bian H, Guo Z, Huang JL, Guo ZN, Yang $\mathrm{P}$, Tang $\mathrm{J}$ and Zhang $\mathrm{JH}$ : Hyperbaric oxygen reduces infarction volume and hemorrhagic transformation through ATP/NAD ${ }^{+} /$Sirtl pathway in hyperglycemic middle cerebral artery occlusion rats. Stroke 48: 1655-1664, 2017.
5. Lanier WL, Hofer RE and Gallagher WJ: Metabolism of glucose, glycogen, and high-energy phosphates during transient forebrain ischemia in diabetic rats: Effect of insulin treatment. Anesthesiology 84: 917-925, 1996.

6. Muranyi M, Fujioka M, He Q, Han A, Yong G, Csiszar K and Li PA: Diabetes activates cell death pathway after transient focal cerebral ischemia. Diabetes 52: 481-486, 2003.

7. Liu WJ, Jiang HF, Rehman FU, Zhang JW, Chang Y, Jing L and Zhang JZ: Lycium barbarum polysaccharides decrease hyperglycemia-aggravated ischemic brain injury through maintaining mitochondrial fission and fusion balance. Int J Biol Sci 13: 901-910, 2017.

8. Lu CJ, Guo YZ, Zhang Y, Yang L, Chang Y, Zhang JW, Jing L and Zhang JZ: Coenzyme Q10 ameliorates cerebral ischemia reperfusion injury in hyperglycemic rats. Pathol Res Pract 213: 1191-1199, 2017.

9. Yang L, Ma YM, Shen XL, Fan YC, Zhang JZ, Li PA and Jing L: The involvement of mitochondrial biogenesis in selenium reduced Hyperglycemia-Aggravated cerebral ischemia injury. Neurochem Res 45: 1888-1901, 2020.

10. Lo EH, Dalkara T and Moskowitz MA: Mechanisms, challenges and opportunities in stroke. Nat Rev Neurosci 4: 399-415, 2003.

11. Zhao Z, Nelson AR, Betsholtz C and Zlokovic BV: Establishment and dysfunction of the Blood-Brain barrier. Cell 163: 1064-1078, 2015.

12. Engelhardt B and Liebner S: Novel insights into the development and maintenance of the blood-brain barrier. Cell Tissue Res 355: 687-699, 2014.

13. Abdullahi W, Tripathi D and Ronaldson PT: Blood-brain barrier dysfunction in ischemic stroke: Targeting tight junctions and transporters for vascular protection. Am J Physiol Cell Physiol 315: C343-C356, 2018.

14. Fang B, Zhang Y and Ma H: Effects of MIR-122a on blood-spinal cord barrier after spinal cord ischemia-reperfusion injury in rats. Chin Pharmacol Bull 33: 703-706, 2017 (In Chinese).

15. Lv J, Hu W, Yang Z, Li T, Jiang S, Ma Z, Chen F and Yang Y: Focusing on claudin-5: A promising candidate in the regulation of BBB to treat ischemic stroke. Prog Neurobiol 161: 79-96, 2018.

16. Miyamori H, Takino T, Kobayashi Y, Tokai H, Itoh Y, Seiki M and Sato H: Claudin promotes activation of pro-matrix metalloproteinase- 2 mediated by membrane-type matrix metalloproteinases. J Biol Chem 276: 28204-28211, 2001.

17. Zhang GS, Tian Y, Huang JY, Tao RR, Liao MH, Lu YM, Ye WF, Wang R, Fukunaga K, Lou YJ and Han F: The $\gamma$-secretase blocker DAPT reduces the permeability of the blood-brain barrier by decreasing the ubiquitination and degradation of occludin during permanent brain ischemia. CNS Neurosci Ther 19: 53-60, 2013.

18. Fanning AS, Jameson BJ, Jesaitis LA and Anderson JM: The tight junction protein ZO-1 establishes a link between the transmembrane protein occludin and the actin cytoskeleton. J Biol Chem 273: 29745-29753, 1998.

19. Sandoval KE and Witt KA: Blood-brain barrier tight junction permeability and ischemic stroke. Neurobiol Dis 32: 200-219, 2008.

20. Paulsson M: Basement membrane proteins: Structure, assembly, and cellular interactions. Crit Rev Biochem Mol Biol 27: 93-127, 1992.

21. Yurchenco PD, Amenta PS and Patton BL: Basement membrane assembly, stability and activities observed through a developmental lens. Matrix Biol 22: 521-538, 2004.

22. Martin GR and Timpl R: Laminin and other basement membrane components. Annu Rev Cell Biol 3: 57-85, 1987.

23. Hallmann R, Horn N, Selg M, Wendler O, Pausch F and Sorokin LM: Expression and function of laminins in the embryonic and mature vasculature. Physiol Rev 85: 979-1000, 2005.

24. Di Russo J, Hannocks MJ, Luik AL, Song J, Zhang X, Yousif L, Aspite G, Hallmann R and Sorokin L: Vascular laminins in physiology and pathology. Matrix Biol 57-58: 140-148, 2017.

25. Hamann GF, Burggraf D, Martens HK, Liebetrau M, Jäger G, Wunderlich N, DeGeorgia M and Krieger DW: Mild to moderate hypothermia prevents microvascular basal lamina antigen loss in experimental focal cerebral ischemia. Stroke 35: 764-769, 2004.

26. Yepes M, Sandkvist M, Wong MK, Coleman TA, Smith E, Cohan SL and Lawrence DA: Neuroserpin reduces cerebral infarct volume and protects neurons from ischemia-induced apoptosis. Blood 96: 569-576, 2000.

27. Abbott NJ, Rönnbäck L and Hansson E: Astrocyte-endothelial interactions at the blood-brain barrier. Nat Rev Neurosci 7: 41-53, 2006.

28. Sofroniew MV and Vinters HV: Astrocytes: Biology and pathology. Acta Neuropathol 119: 7-35, 2010. 
29. Dodson RF, Chu LW, Welch KM and Achar VS: Acute tissue response to cerebral ischemia in the gerbil. An ultrastructural study. J Neurol Sci 33: 161-170, 1977.

30. Kamada $\mathrm{H}, \mathrm{Yu} F$, Nito $\mathrm{C}$ and $\mathrm{Chan} \mathrm{PH}$ : Influence of hyperglycemia on oxidative stress and matrix metalloproteinase-9 activation after focal cerebral ischemia/reperfusion in rats: Relation to blood-brain barrier dysfunction. Stroke 38: 1044-1049, 2007.

31. Zhang S, Shao SY, Song XY, Xia CY, Yang YN, Zhang PC and Chen NH: Protective effects of Forsythia suspense extract with antioxidant and anti-inflammatory properties in a model of rotenone induced neurotoxicity. Neurotoxicology 52: 72-83, 2016.

32. Zhang S, An Q, Wang T, Gao S and Zhou G: Autophagyand MMP-2/9-mediated reduction and redistribution of ZO-1 contribute to Hyperglycemia-increased Blood-Brain barrier permeability during early reperfusion in stroke. Neuroscience 377: 126-137, 2018.

33. Jiang X, Andjelkovic AV, Zhu L, Yang T, Bennett MVL, Chen J, Keep RF and Shi Y: Blood-brain barrier dysfunction and recovery after ischemic stroke. Prog Neurobiol 163-164: 144-171, 2018.

34. Gelderblom M, Leypoldt F, Steinbach K, Behrens D, Choe CU, Siler DA, Arumugam TV, Orthey E, Gerloff C, Tolosa E and Magnus T: Temporal and spatial dynamics of cerebral immune cell accumulation in stroke. Stroke 40: 1849-1857, 2009.

35. Jickling GC, Liu D, Ander BP, Stamova B, Zhan X and Sharp FR Targeting neutrophils in ischemic stroke: Translational insights from experimental studies. J Cereb Blood Flow Metab 35: 888-901, 2015.

36. da Fonseca AC, Matias D, Garcia C, Amaral R, Geraldo LH, Freitas $\mathrm{C}$ and Lima FR: The impact of microglial activation on blood-brain barrier in brain diseases. Front Cell Neurosci 8: 362 , 2014.

37. Hu X, De Silva TM, Chen J and Faraci FM: Cerebral vascular disease and neurovascular injury in ischemic stroke. Circ Res 120 449-471, 2017

38. Fan Q, Chen M, Zuo L, Shang X, Huang MZ, Ciccarelli M Raake P, Brinks H, Chuprun KJ, Dorn GW II, et al: Myocardial ablation of G Protein-Coupled Receptor Kinase 2 (GRK2) decreases Ischemia/Reperfusion injury through an Anti-Intrinsic apoptotic pathway. PLoS One 8: e66234, 2013.

39. Morrison HW and Filosa JA: A quantitative spatiotemporal analysis of microglia morphology during ischemic stroke and reperfusion. J Neuroinflammation 10: 4, 2013.

40. Thorsten W and Hans-Gerd L: IJBlob: An ImageJ Library for Connected Component Analysis and Shape Analysis. J Open Res Softw 1: p.e6, 2013.

41. Otxoa-de-Amezaga A, Miro-Mur F, Pedragosa J, Gallizioli M, Justicia C, Gaja-Capdevila N, Ruíz-Jaen F, Salas-Perdomo A, Bosch A, Calvo M, et al: Microglial cell loss after ischemic stroke favors brain neutrophil accumulation. Acta Neuropathol 137: 321-341, 2019.

42. Tyson GW, Teasdale GM, Graham DI and McCulloch J: Focal cerebral ischemia in the rat: Topography of hemodynamic and histopathological changes. Ann Neurol 15: 559-567, 1984.

43. Sweeney MD, Zhao Z, Montagne A, Nelson AR and Zlokovic BV: Blood-Brain barrier: From physiology to disease and back. Physiol Rev 99: 21-78, 2019.

44. Kang $M$ and Yao Y: Basement membrane changes in ischemic stroke. Stroke 51: 1344-1352, 2020.

45. Kacem K, Lacombe P, Seylaz J and Bonvento G: Structural organization of the perivascular astrocyte endfeet and their relationship with the endothelial glucose transporter: A confocal microscopy study. Glia 23: 1-10, 1998

46. Sasaki Y, Ohsawa K, Kanazawa H, Kohsaka S and Imai Y: Iba1 is an actin-cross-linking protein in macrophages/microglia. Biochem Biophys Res Commun 286: 292-297, 2001.

47. Yang C, Hawkins KE, Dore S and Candelario-Jalil E: Neuroinflammatory mechanisms of blood-brain barrier damage in ischemic stroke. Am J Physiol Cell Physiol 316: C135-C153, 2019.

48. Barrett-Connor E and Khaw KT: Diabetes mellitus: An independent risk factor for stroke? Am J Epidemiol 128: 116-123, 1988.

49. Luitse MJ, Biessels GJ, Rutten GE and Kappelle LJ: Diabetes, hyperglycaemia, and acute ischaemic stroke. Lancet Neurol 11: 261-271, 2012.

50. Ankolekar S, Rewell S, Howells DW and Bath PM: The influence of stroke risk factors and comorbidities on assessment of stroke therapies in humans and animals. Int J Stroke 7: 386-397, 2012.

51. van Sloten TT, Sedaghat S, Carnethon MR, Launer LJ and Stehouwer CDA: Cerebral microvascular complications of type 2 diabetes: Stroke, cognitive dysfunction, and depression. Lancet Diabetes Endocrinol 8: 325-336, 2020.
52. Sander D and Kearney MT: Reducing the risk of stroke in type 2 diabetes: Pathophysiological and therapeutic perspectives. J Neurol 256: 1603-1619, 2009.

53. Ergul A,Kelly-Cobbs A, Abdalla Mand Fagan SC: Cerebrovascular complications of diabetes: Focus on stroke. Endocr Metab Immune Disord Drug Targets 12: 148-158, 2012.

54. Reeves MJ, Vaidya RS, Fonarow GC, Liang L, Smith EE, Matulonis R, Olson DM and Schwamm LH: Quality of care and outcomes in patients with diabetes hospitalized with ischemic stroke: Findings from get with the Guidelines-Stroke. Stroke 41: e409-e417, 2010

55. Warner DS, Smith ML and Siesjö BK: Ischemia in normo-and hyperglycemic rats: Effects on brain water and electrolytes. Stroke 18: 464-471, 1987.

56. Godínez-Rubí M, Rojas-Mayorquín AE and Ortuño-Sahagún D: Nitric oxide donors as neuroprotective agents after an ischemic stroke-related inflammatory reaction. Oxid Med Cell Longev 2013: 297357, 2013

57. Fukuta T, Ishii T, Asai T, Sato A, Kikuchi T, Shimizu K, Minamino T and Oku N: Treatment of stroke with liposomal neuroprotective agents under cerebral ischemia conditions. Eur J Pharm Biopharm 97(Pt A): 1-7, 2015.

58. Birmingham K: Future of neuroprotective drugs in doubt. Nat Med 8: 5, 2002

59. Guo S, Kim WJ, Lok J, Lee SR, Besancon E, Luo BH, Stins MF, Wang X, Dedhar S and Lo EH: Neuroprotection via matrix-trophic coupling between cerebral endothelial cells and neurons. Proc Natl Acad Sci USA 105: 7582-7587, 2008.

60. Iadecola C: Neurovascular regulation in the normal brain and in Alzheimer's disease. Nat Rev Neurosci 5: 347-360, 2004.

61. Jia W, Lu R, Martin TA and Jiang WG: The role of claudin-5 in blood-brain barrier (BBB) and brain metastases (review). Mol Med Rep 9: 779-785, 2014.

62. Findley MK and Koval M: Regulation and roles for claudin-family tight junction proteins. IUBMB Life 61: 431-437, 2009.

63. Van Itallie CM and Anderson JM: Claudin interactions in and out of the tight junction. Tissue Barriers 1: e25247, 2013.

64. Anderson JM and Van Itallie CM: Physiology and function of the tight junction. Cold Spring Harb Perspect Biol 1: a002584, 2009.

65. Sladojevic N, Stamatovic SM, Keep RF, Grailer JJ, Sarma JV, Ward PA and Andjelkovic AV: Inhibition of junctional adhesion molecule-A/LFA interaction attenuates leukocyte trafficking and inflammation in brain ischemia/reperfusion injury. Neurobiol Dis 67: 57-70, 2014.

66. Abbruscato TJ, Lopez SP, Mark KS, Hawkins BT and Davis TP: Nicotine and cotinine modulate cerebral microvascular permeability and protein expression of ZO-1 through nicotinic acetylcholine receptors expressed on brain endothelial cells. J Pharm Sci 91: 2525-2538, 2002.

67. Fischer S, Wobben M, Marti HH, Renz D and Schaper W: Hypoxia-Induced hyperpermeability in brain microvessel endothelial cells involves VEGF-Mediated changes in the expression of Zonula Occludens-1. Microvasc Res 63: 70-80, 2002.

68. Mark KS and Davis TP: Cerebral microvascular changes in permeability and tight junctions induced by hypoxia-reoxygenation. Am J Physiol Heart Circ Physiol 282: H1485-H1494, 2002.

69. Wang H, Li T, Zhao L, Sun M, Jian Y, Liu J, Zhang Y, Li Y, Dang $M$ and Zhang G: Dynamic effects of ioversol on the permeability of the blood-brain barrier and the expression of ZO-1/occludin in rats. J Mol Neurosci 68: 295-303, 2019.

70. Bazzoni G and Dejana E: Endothelial cell-to-cell junctions: Molecular organization and role in vascular homeostasis. Physiol Rev 84: 869-901, 2004.

71. Huber JD, Egleton RD and Davis TP: Molecular physiology and pathophysiology of tight junctions in the blood-brain barrier Trends Neurosci 24: 719-725, 2001.

72. Xu L, Nirwane A and Yao Y: Basement membrane and blood-brain barrier. Stroke Vasc Neurol 4: 78-82, 2018.

73. Yao Y: Basement membrane and stroke. J Cereb Blood Flow Metab 39: 3-19, 2019.

74. Kwon I, Kim EH, del Zoppo GJ and Heo JH: Ultrastructural and temporal changes of the microvascular basement membrane and astrocyte interface following focal cerebral ischemia. J Neurosci Res 87: 668-676, 2009.

75. Kazmierski R, Michalak S, Wencel-Warot A and Nowinski WL: Serum tight-junction proteins predict hemorrhagic transformation in ischemic stroke patients. Neurology 79: 1677-1685, 2012.

76. Khatri R, McKinney AM, Swenson B and Janardhan V Blood-brain barrier, reperfusion injury, and hemorrhagic transformation in acute ischemic stroke. Neurology 79 (13 Suppl 1): S52-S57, 2012. 
77. Rodríguez-Arellano J, Parpura V, Zorec R and Verkhratsky A: Astrocytes in physiological aging and Alzheimer's disease. Neuroscience 323: 170-182, 2016.

78. Hansson E and Rönnbäck L: Astrocytes in glutamate neurotransmission. FASEB J 9: 343-350, 1995.

79. Jing L, He Q, Zhang JZ and Li PA: Temporal profile of astrocytes and changes of oligodendrocyte-based myelin following middle cerebral artery occlusion in diabetic and non-diabetic rats. Int J Biol Sci 9: 190-199, 2013.

80. Anderson MA, Burda JE, Ren Y, Ao Y, O'Shea TM, Kawaguchi R, Coppola G, Khakh BS, Deming TJ and Sofroniew MV: Astrocyte scar formation aids central nervous system axon regeneration. Nature 532: 195-200, 2016.

81. Overman JJ, Clarkson AN, Wanner IB, Overman WT, Eckstein I, Maguire JL, Dinov ID, Toga AW and Carmichael ST: A role for ephrin-A5 in axonal sprouting, recovery, and activity-dependent plasticity after stroke. Proc Natl Acad Sci USA 109: E2230-E2239, 2012.

82. Liauw J, Hoang S, Choi M, Eroglu C, Choi M, Sun GH, Percy M, Wildman-Tobriner B, Bliss T, Guzman RG, et al: Thrombospondins 1 and 2 are necessary for synaptic plasticity and functional recovery after stroke. J Cereb Blood Flow Metab 28: 1722-1732, 2008.
83. Fitch MT and Silver J: CNS injury, glial scars, and inflammation: Inhibitory extracellular matrices and regeneration failure. Exp Neurol 209: 294-301, 2008

84. Bannister JV, Bellavite P, Davoli A, Thornalley PJ and Rossi F: The generation of hydroxyl radicals following superoxide production by neutrophil NADPH oxidase. FEBS Lett 150: 300-302, 1982.

85. McColl BW, Rothwell NJ and Allan SM: Systemic inflammatory stimulus potentiates the acute phase and CXC chemokine responses to experimental stroke and exacerbates brain damage via interleukin-1- and neutrophil-dependent mechanisms. J Neurosci 27: 4403-4412, 2007.

86. Gautier S, Ouk T, Petrault O, Caron J and Bordet R: Neutrophils contribute to intracerebral haemorrhages after treatment with recombinant tissue plasminogen activator following cerebral ischaemia. Br J Pharmacol 156: 673-679, 2009.

87. Pan W and Kastin AJ: Tumor necrosis factor and stroke: Role of the blood-brain barrier. Prog Neurobiol 83: 363-374, 2007.

(i) $($ ) This work is licensed under a Creative Commons

Attribution-NonCommercial-NoDerivatives 4.0 International (CC BY-NC-ND 4.0) License. 